Canadian University Music Review

Canadian University Music Review

Revue de musique des universités canadiennes

\title{
Les Six Études Pour Piano de György Ligeti ou l'Art Subtil de Créer en Assumant les Référents Culturels
}

Denys Bouliane

Volume 9, numéro 2, 1989

URI : https://id.erudit.org/iderudit/1014906ar

DOI : https://doi.org/10.7202/1014906ar

Aller au sommaire du numéro

Éditeur(s)

Canadian University Music Society / Société de musique des universités canadiennes

ISSN

0710-0353 (imprimé)

2291-2436 (numérique)

Découvrir la revue

Citer cet article

Bouliane, D. (1989). Les Six Études Pour Piano de György Ligeti ou l'Art Subtil de Créer en Assumant les Référents Culturels. Canadian University Music Review / Revue de musique des universités canadiennes, 9(2), 36-83.

https://doi.org/10.7202/1014906ar

All Rights Reserved ( C Canadian University Music Society / Société de musique des universités canadiennes, 1989
Ce document est protégé par la loi sur le droit d'auteur. L'utilisation des services d'Érudit (y compris la reproduction) est assujettie à sa politique d'utilisation que vous pouvez consulter en ligne.

https://apropos.erudit.org/fr/usagers/politique-dutilisation/ 


\section{LES SIX ÉTUDES POUR PIANO \\ DE GYÖRGY LIGETI \\ OU L'ART SUBTIL DE CRÉER \\ EN ASSUMANT \\ LES RÉFÉRENTS CULTURELS}

\section{Denys Bouliane}

Le texte que voici est l'adaptation d'une émission écrite pour la radio allemande DeutschlandFunk à l'automne 1987 et diffusée par cette même radio le 27 avril 1988. Je remercie la maison d'édition Schott d'avoir autorisé, spécialement pour cet article, la photographie de plusieurs extraits des Études de Ligeti.

D. B.

Ce n'est un secret pour personne que l'évolution de la musique nouvelle $s^{\prime}$ est faite, depuis une quarantaine d'années, plutôt "en marge" du grand public mélomane. On peut, évidemment, en accuser notre infrastructure musicale, qui a tendu de plus en plus à un marketing conservateur des "valeurs sûres"... mais, tout de même... il faudrait être naïf pour ne pas réaliser que, dans bien des cas, la tâche de nos vaillants compositeurs d'avant-garde semble avoir été de formaliser l'espace sonore musical en ne s'attachant prioritairement qu'à la pure ordonnance du matériau.... Le compositeur comme simple "assembleur de sons" ? Peut-être y aurait-il eu une confusion plus ou moins consciemment entretenue entre le fait de jongler avec intelligence et originalité avec une grammaire et une syntaxe musicales à valeur perceptuelle et, d'autre part, l'élaboration de règles compositionnelles phénoménologiquement arbitraires, auxquelles le compositeur s'amuse à adhérer ou à se soustraire selon son bon plaisir...

En fait, l'un des problèmes fondamentaux de la composition contemporaine est la difficulté d'inventer des FONCTIONNALITÉS psychologiquement et psychoacoustiquement pertinentes. ${ }^{1}$ Schœnberg

1 Le problème est d'autant plus complexe que le compositeur a eu et a à œuvrer dans un nombre sans cesse croissant de "dimensions musicales" dignes d'intérêt. 
(1975) disait à peu près ceci : n'importe quel événement apparaissant en un point quelconque de l'espace musical provoque un effet non limité à son aire immédiate; autrement dit, il n'agit pas seulement sur un plan spécifique, mais dans toutes les directions et sur tous les plans. Á un niveau encore plus global, on pourrait paraphraser Schœnberg en déclarant que : $n^{\prime}$ importe quel événement musical dans une composition donnée a une portée culturelle signifiante non limitée à la seule œuvre dont il est tiré; autrement dit, cet événement musical ne peut être "culturellement neutre" et complètement dissocié du contexte dont il est issu; le geste musical réfère à des habitudes d'écoute conditionnées par la nature même de l'appareil perceptuel et, pour une bonne part, par le bagage culturel accumulé dans la tradition.

Il peut être relativement aisé d'assembler des sons sur le papier et d'aboutir, par le jeu de combinatoires savantes, à une forme de "complexité pour l'œil". Toutefois, le travail avec la matière musicale est une entreprise autrement subtile et raffinée, car il suppose une connaissance des mécanismes perceptuels humains, de la psychologie de la perception ainsi que des implications référentielles culturelles du matériau et des structures élaborés. Que cette connaissance soit empirique ou acquise ne change rien au fait que le compositeur, qu'il le veuille ou non, est constamment confronté au problème de la "communicabilité" de son esthétique - si nouvelle soit-elle -, et le fait de s'imaginer pouvoir réaliser une tabula rasa et de recommencer à zéro relève d'une métaphore séduisante plutôt que de l'expérience musicale concrète.

Il a tout de même été donné à quelques compositeurs, depuis une quarantaine d'années, de trouver un moyen efficace et raffiné de communiquer une pensée musicale et cela, sans remâcher et régurgiter sempiternellement les syntaxes et schèmes directement livrés par la tradition. Un "cas" particulièrement intéressant - si j'ose m'exprimer ainsi - est celui de György Ligeti. Que, dès le début des années 60, sa musique ait été rapidement associée aux mouvements d'avant-garde a probablement contribué à masquer deux faits importants : d'une part, "le style Ligeti" n'a que très peu à voir avec nombre de spéculations théoriques des années 50 et 60 ; d'autre part, bien loin de nier les schèmes d'écoute façonnés au cours de l'évolution de la musique occidentale, le compositeur a su les utiliser de manière incroyablement efficace. Une œuvre comme Atmosphères (1961), par exemple, se réfère directement à l'habitus de la musique symphonique romantique, un peu comme si sa gestuelle pure nous en était présentée "figée" dans l'espace musical. C'est là une manière géniale de se référer constamment à la tadition sans jamais la citer explicitement. Loin de faire table rase, Ligeti réalisait 
ainsi une sorte de distorsion de perspective en mettant prioritairement l'accent sur des phénomènes acoustiques extrêmement "prégnants", tels les processus de transformation de couleur orchestrale, de densité, de dynamique, de vitesse, les jeux de rapprochement et d'éloignement illusoires de la source sonore, de transfert et de contraste des tessitures. On peut retrouver tous ces éléments dans le répertoire orchestral romantique et post-romantique, mais ici, ils sont "hyper-grossis" et deviennent moteurs d'un discours faisant directement appel à notre perception sensible.

Tout au long de la trajectoire compositionnelle de Ligeti couvrant le début des années 60 à la fin des années 70 , on pourrait retracer une forme particulièrement originale de "ré-émergence" de référents culturels; le compositeur y a toujours fait allusion de façon plus ou moins voilée et leur maniement conscient a contribué à asseoir son langage sur des bases phénoménologiques hautement probantes. Ainsi, au fil des cuvres, de Lontano (1967), en passant par le Deuxième Quatuor à cordes (1968) et Melodien (1971), jusqu'à San Francisco Polyphony (1976) et aux Trois pièces pour deux pianos : Monument-Selbstportrait-Bewegung (1976), le travail des constellations intervalliques, les techniques de polarisation harmonique, le traitement contrapuntique extrêmement raffiné, les structures rythmiques et métriques, de même que les références formelles aux divers genres traditionnels ont graduellement - j'oserais même dire "tout naturellement" - refait surface. Tous ces éléments de langage apparaissent toutefois réactualisés ${ }^{2}$ et dans une conception qui tient autant $\mathrm{du}$ système tonal que de la pensée sérielle. Il suffirait de mentionner, par exemple, la directionnalité du discours : le principe d'attraction par entonnoir, où le total chromatique est dirigé inéluctablement sur des pôles d'attraction précis ou alors, "happé" par une seule hauteur privilégiée. $D^{\prime}$ autre part, le système de permutations constantes, l'idée de laisser un processus - une mécanique - s'épuiser (ou de le conduire subtilement à son épuisement), ainsi que la fusion des divers paramètres musicaux ont beaucoup en commun avec la pensée sérielle. Tout cela concourt à renforcer l'impression que, chez Ligeti, les référents culturels apparaissent comme potentiellement rapprochés ou éloignés, qu'il s'agisse de Monteverdi, Bartók, Palestrina, Mahler, Nancarrow ou Reich.

$2 \mathrm{Ou}$, comme le disent si bien les Américains, "revisités". 
Un trait important de la personnalité de Ligeti est ce désir insatiable de communiquer et de "réagir" en tant qu'artiste aux multiples stimuli socio-culturels. En ce sens, l'attitude du compositeur est tout à l'opposé $\mathrm{du}$ dogmatisme structurel. Comme plusieurs commentateurs l'ont souvent souligné, il est un des rares compositeurs de sa génération et de sa stature n'ayant jamais envisagé de "faire école". Il a toujours voulu se réserver le droit de "patauger à pleines mains" dans l'humus culturel, et les multiples référents que $1^{\prime}$ on rencontre et devine dans sa musique sont assumés pour leur potentiel référentiel même; ils se fondent en une sorte de témoignage esthétique fécondé et fécondant à son tour, grâce auquel l'auditeur est constamment suspendu aux frontières du connu et du possible. Il ne s'agit pas simplement pour Ligeti de construire un langage de toutes pièces mais bien plutôt de réaliser, par maintes transplantations nerveuses, transfusions sanguines et greffes in vivo, un organisme musical nouveau, se nourrissant aux sources même de ses prélèvements et intégrant dans son métabolisme l'énergie de ses ingestions. Loin d'exiger la suspension ou le détournement des habitudes d'écoute, la musique du compositeur les présuppose. Ligeti s'oblige à demeurer à l'écoute de son époque et à réévaluer constamment la pertinence et la communicabilité de son propos.

Ainsi, comme Apparitions (1958-59) et Atmosphères (1961) pouvaient constituer une "réaction" dans le contexte idéel de cette époque, les nouvelles œuvres des années 80 , tout en prolongeant la démarche personnelle de Ligeti, apportent un "son de cloche" extrêmement pertinent dans une période où déferle une grande vague "restaurative" - souvent pratiquement pastichisante - et où le dogmatisme conceptuel de l'avant-garde européenne des années 60 s'étiole en épigones... Bien sûr, les dernières œuvres de Ligeti ont quelque chose de "classique", de par leur conception même, et il ne faudrait pas y chercher un quelconque caractère "expérimental", caractère que la musique du compositeur n'a, de toute manière, que fort épisodiquement adopté. ${ }^{3}$ Cet aspect "classique" ne saurait toutefois faire oublier un élément capital pour la compréhension et l'appréciation d'œuvres telles que le Trio pour violon, cor et piano (1982), le Concerto pour piano (commencé en 1985) ou les Six Études pour piano (1985) : dans ces musiques se perpétuent, mais aussi se revitalisent plusieurs des éléments les plus riches de la tradition musicale européenne. En outre, la musique de Ligeti se trouve

3 Le Poème symphonique pour 100 métronomes (1962) et, peut-être aussi, certaines sections "collagistes" du Grand Macabre (1975) en seraient des exemples. 
actuellement au carrefour d'un grand nombre de traditions débordant les cadres de la musique de concert occidentale; elle lorgne tantôt du côté de la musique des Balkans, elle puise des éléments essentiels dans la musique des cultures africaines subsahariennes, elle "flirte" à l'occasion avec la gestuelle et la vivacité rythmique des musiques brésiliennes et caraïbéennes. Certes, une attitude "syncrétique", mais aussi - comme on le constatera avec l'analyse des Six Études pour piano - technique subtile de "rappels" référentiels toujours présents, tantôt latents, tantôt émergents, sollicitant et remettant constamment en jeu notre mémoire culturelle.

\section{Les SIX ÉTUDES POUR PIANO ${ }^{4}$}

Deux points à préciser avant d'entrer dans le propos. D'une part, la première Étude sera scrutée beaucoup plus en profondeur que les suivantes, lesquelles seront plutôt commentées de manière panoramique. D'autre part, étant donné l'importance accordée, dans l'analyse qui suit, à l'enjeu sonore des œuvres discutées, et bien que le lecteur puisse disposer de transcriptions et exemples musicaux imprimés, l'audition des pièces citées est fortement recommandée. À cette fin, on trouvera en notes infrapaginales des informations relatives à plusieurs gravures commercialement disponibles sur disque ou cassette.

Étude 1 : DÉSORDRE (voir exemple 1)

Après une première audition, essayons de préciser sommairement l'impression sonore dégagée par cette Étude :

1. de manière très générale, le sentiment d'un champ harmonique en mouvance constante, quoique dans une palette de couleurs très réduite;

2. une forme d'ambiguité entre ce que l'on perçoit comme l'avant-plan et l'arrière-plan sonores;

3. un aspect de redondance brisée - de répétition "tronquée";

4. la sensation d'une grande mobilité rythmique et métrique;

4 La partition (fac-similé) est disponible chez Schott (ED/7428) depuis la fin de 1986. Un enregistrement par le pianiste Volker Banfield a été réalisé sur étiquette Wergo (60134/LP). 
5. le sentiment d'un déplacement continu et dirigé dans l'espace des tessitures.

En ce qui concerne la couleur harmonique, notre impression se trouve rapidement confirmée par un coup d'œil à la partition (cf. les premières mesures de l'exemple 1): très simplement, la main droite du pianiste se meut exclusivement sur les touches blanches du clavier et le contour mélodique tend à suggérer, à tout le moins au départ, un mode locrien (sur si); la main gauche, elle, n'évoluera que sur les touches noires, soit dans un mode pentatonique. Avec la superposition des deux mains, on aboutit au total chromatique. En fait, au-delà du pur aspect "pianistiquement idiomatique" de ce procédé, la référence à la pensée polymodale d'un Bartók est ici évidente. On se rappellera que Bartók, autour des années 1900, se démarque de plus en plus de la tradition musicale romantique des maîtres allemands et se met à élaborer un nouveau langage mélodico-harmonique puisant dans le riche bagage des cultures populaires hongroise et roumaine. On note, entre autres, l'assimilation de l'échelle pentatonique de la musique hongroise et des modes dorien, phrygien et mixolydien de la musique roumaine. Vers 1907-1908, Bartók utilisera de plus en plus la superposition de modes dans une même pièce (bi ou poly-modalité). On pense, notamment, à la quatrième des 14 Bagatelles pour piano, op. 6 , où la main droite est écrite avec une armature de 4 dièzes et la main gauche, avec 4 bémols. Bartók concevra aussi la possibilité d'aboutir au total chromatique en superposant les modes lydien et phrygien sur une même tonique. $C^{\prime}$ est précisément à ce type de construction polymodale que fait penser la première Étude de Ligeti. En réalité, il ne s'agit pas ici de modes "fonctionnels" au plan harmonique, mais la référence en tant que "couleur" ou "qualité harmonique" est intéressante; de plus, il ne faut pas oublier que Ligeti a toujours travaillé les constellations d'intervalles d'une manière extrêmement raffinée, en se souciant de la qualité, de la couleur des structures intervalliques privilégiées à l'intérieur de la toile de fond du total chromatique. Ici, dans cette première Étude, il s'agit de ce que j'appellerais "un cas particulier", lequel, de par sa nature même., joue le rôle d'un référent culturel très accusé.

Abordons maintenant les notions d'avant-plan, de plan moyen et d'arrière-plan, qui ont toujours joué un rôle important chez Ligeti. Le compositeur a utilisé de mille et une façons la possibilité de faire lentement émerger de textures complexes des structures intervalliques, des contours et des mélodies privilégiés; des œuvres comme Lontano ou Melodien sont exemplaires à cet effet. $D^{\prime}$ autre part, dans une pièce comme Continuum (1968), Ligeti réussit de véritables trompe-l'oreille en jouant avec ce que l'on pourrait appeler des super-signaux : d'une trame 
en mouvement très rapide émergent des structures mélodiques et rythmiques engendrées par la répétition plus ou moins régulière de certains sons privilégiés. Il en va de manière similaire pour Monument, première des Trois pièces pour deux pianos (1976); Monument est en effet entièrement basé sur l'apparition de patterns inhérents, qui sont créés par la juxtaposition polymétrique de plusieurs "terrasses" à dynamique fixe. Les patterns sont ici issus de l'interconnection auditive de plusieurs couches musicales indépendantes : notre oreille perçoit les diverses strates superposées, mais notre cerveau en réalise une fusion en les combinant en patterns résultants. Les références à la musique du compositeur mexicano-américain Conlon Nancarrow sont manifestes (on pense, en particulier, à Study no 20), bien que Ligeti n'ait pas eu connaissance de cette musique à l'époque de la composition de Monument. Toutefois, c'est en pleine connaissance de la musique d'un Nancarrow et, plus important encore, c'est fasciné par les musiques polyrythmiques des cultures subsahariennes que Ligeti a entrepris la composition de ses Six Études de même que de son Concerto pour piano.

Le musicologue Gerhard Kubik a très bien su décrire ce phénomène de patterns inhérents : "Il s'agit de structures mélodico-rythmiques organisées et perceptibles en tant que telles, qui semblent 'surgir' de l'image globale d'un passage musical, telles des illusions acoustiques. Ces structures mélodiques ne sont pas jouées comme telles par les musiciens mais sont toutefois le plus souvent consciemment élaborées." $(1983: 347)^{5}$ Un très bel exemple serait celui de la musique Akadinda, jouée sur un même xylophone par un ensemble de six musiciens de Salama, en Ouganda : voir exemple 2, tiré de Kubik (1983: 384). ${ }^{6}$

Les musiciens s'assoient face à face de chaque côté de l'instrument et chaque groupe joue en octaves parallèles l'une de deux mélodies de base. Ces deux mélodies s'imbriquent comme deux roues d'engrenage dans un tempo d'environ 200 pulsations (d.) par minute. Le résultat combi né nous donne une suite de patterns en intervalles irréguliers se déplaçant à une vitesse vertigineuse (600 pulsations par minute); cette suite de patterns se répétera comme une boucle en fonction de la longueur des deux mélodies de base.

[ Ibidem : 384 ]

5 La traduction des citations de Kubik est mienne. (D.B.)

6 Référence pour audition : "Blind Musicians of Salama", dans Musik in Afrika, cassettes d'accompagnement, ex. 55 . 
De manière analogue, la quatrième (Fanfares), la sixième (Automne à Varsovie), tout autant que la première des Six Études de Ligeti sont basées sur la superposition - et l'interaction, au niveau de la perception - de plusieurs strates musicales. Dans Désordre, on peut aisément distinguer deux plans sonores principaux, qui se dédoubleront en cours de morceau : au premier plan, une mélodie accentuée en octaves, et, en arrière-plan, une ligne diatonique légèrement brisée, à tournure ascendante, qui "remplit" en quelque sorte l'espace modal. Les lignes mélodiques de l'arrière-plan correspondent en principe à l'articulation de la mélodie en octaves mais peuvent aussi se développer de façon autonome, "enjambant" la mélodie principale. (Voir les 14 premières mesures de Désordre, la main droite en particulier, à l'exemple 1). La même idée est reprise pour la main gauche, mais en mode pentatonique. ${ }^{7}$ Fait intéressant : comme les 7 degrés du mode de si de la main droite sont alors réduits à 5 , le contour de la mélodie se trouvera forcément transformé et la fréquence des notes répétées sera ainsi plus grande. (Voir exemple 3).

Ce type de mélodie $n^{\prime}$ est pas sans rappeler un trait typiquement hongrois, tel qu'assimilé par Bartók : le motif principal est constitué par la répétition de la première note de la mélodie, sorte de "motif de tête" rythmiquement insistant. Il y a là, possiblement, une relation avec la langue hongroise même : en effet, le hongrois - tout comme le tchécoslovaque - accentue de manière prioritaire la première syllabe de chaque mot. Qu'il suffise, à titre d'illustration, de se rappeler le début de l'Allegro barbaro de Bartók (voir exemple 4), ou encore, le thème principal de la Sonate pour deux pianos et percussions (voir exemple 5) : c'est un peu comme si la "qualité rythmique" du motif définissait le caractère même de la mélodie.

Revenons maintenant à Ligeti, pour examiner d'un peu plus près la structure de la mélodie de Désordre et découvrir comment son traitement conduit à cette impression de redondance brisée dont il a déjà été question. La mélodie complète se subdivise en 3 éléments, chacun débutant par le "motif de tête" (voir exemple 6). Nous avons ainsi un premier élément $a$, de 4 mesures; puis, un deuxième élément $a^{\prime}$, également de 4 mesures, constituant une sorte de séquence; et, enfin, un troisième élément $b$, de 6 mesures, qui développe l'idée de $a$ et "remplit" l'espace modal heptatonique.

7 On se rappellera que la main gauche ne joue que sur les touches noires du clavier. 
Un thème très "classique" donc dans sa conception, rappelant une des coupes formelles les plus typiques de l'époque classique, la sentence, telle que décrite par Schœenberg (1967). ${ }^{8}$ Un exemple parmi tant d'autres : le thème principal de la Sonate op. 2 no 3 de Beethoven (voir exemple 7). Un modèle de 2 mesures (tonique vers dominante) est suivi de sa forme "séquencée" (dominante vers tonique), puis d'un développement de 4 mesures, lui-même basé sur un principe de répétition variée élaborée.

Le thème de coupe classique de Ligeti servira de "pierre à bâtir" pour toute $l^{\prime}$ Etude et sera incessamment répété, mais chaque fois transposé d'un degré dans l'échelle locrienne (mode de si), couvrant le registre moyen jusqu'à l'extrême aigu.

Jetons maintenant un coup d'oeil sur l'articulation métrique. Le modèle mélodique de 4 mesures repose sur une accentuation asymétrique des 8 pulsations rapides de chaque mesure : la formule type est $3+5$ et son inversion, $5+3$. Ce type d'articulation est, comme on le sait, assez fréquent dans toute la musique des Balkans, mais plus particulièrement encore, dans la tradition musicale bulgare. Pour une courte illustration très typique, voir exemple $8:$ il s'agit de la danse Gankino Horo, jouée par un ensemble de musiciens de Sofia; ${ }^{9}$ nous avons dans ce cas un nombre impair de pulsations, 11 , qui est articulé en $2+2+3+2+2$ et parfois $2+3+2+2+2$.

Cette technique bien connue a été reprise par de nombreux compositeurs hongrois au cours de la première moitié du XXe siècle; les exemples abondent dans la musique de Kodály, Lajtha, Kadosa, Veress et, plus récemment, dans celle de György Kurtág. Le Scherzo "Alla bulgarese" du Quatuor no 5 de Bartók en est bel exemple, avec son accentuation en $4+2+3$ (voir exemple 9).

Dans ses travaux sur la musique des Balkans, l'ethnomusicologue roumain Constantin Brailoiu parle de rythme Aksak pour ce type d'articulation asymétrique. Fait intéressant, le musicologue tunisien Salah el Mahdi (1972) définit l'Aksak comme l'un des multiples modes rythmiques arabes, particulièrement fréquent en Turquie mais également présent dans tout le Maghreb : sa structure de 9 pulsations s'articule en $2+2+3+2$. Cette pensée en subdivisions asymétriques de mesures aux nombres pairs ou impairs de pulsations constitue, certes, un lien

8 Voir les chapitres V et VIII.

9 Cf. Folk Music of Bulgaria, Topic Records, London (12 T 107). 
intéressant entre la musique des Balkans, d'une part, et les traditions arabes maghrébines, syrio-égyptiennes et irakiennes, $d$ 'autre part. De ce point de vue, la musique polyrythmique africaine subsaharienne semble procéder d'une conception différente ; quoique cette musique utilise les "apparentes" divisions asymétriques du nombre de pulsations favorisant les structures de 8 pulsations (articulation typique en $3+5$ ), de $12(5+7)$, mais aussi de $16(7+9)$ et $24(11+13)-$, elle ne peut être directement considérée comme métrique, puisqu'il s'agit plutôt d'une pensée en patterns additifs, d'après les plus récentes études musicologiques de Artur Simon, Gerhard Kubik ou Alfons M. Dauer. ${ }^{10}$ Souvent, il n'existe pas de "mesure" dans le sens occidental du terme, avec la hiérarchisation de temps forts et de temps faibles; les musiciens penseraient plutôt en additionnant des pulsations, comme $2+3+2$ etc. Ce qui est particulier, c'est la superposition de plusieurs patterns rythmiques de structure et de longueur différentes, tous réunis par un dénominateur commun très rapide (en allemand : Nennwert) On aboutit, dans certains cas, à une polyrythmie très complexe, et l'oreille occidentale a souvent tendance à interpréter ces superpositions comme des ambiguités métriques, puisque divers multiples de 2 ou 3 peuvent être simultanément ressentis comme "temps" sous-jacents.

Un exemple assez simple serait le lied populaire $E$ Juba malik aleya ana (Oh Juba, qu'est-ce qui ne va pas?), tel que chanté par un groupe de femmes et $d^{\prime}$ hommes de Geigar, au Soudan (voir exemple 10). ${ }^{11} \mathrm{La}$ mélodie elle-même, chantée à l'unisson, peut être interprétée "métriquement" de plusieurs manières; l'une des possibilités est la combinaison $3+3+2+2+2+3+3$, pour un total de 18 pulsations ( $h$. La mélodie est accompagnée de claquements de mains par deux proupes d'exécutants : d'une part, les hommes battant une pulsation régulière en $[2+2+2]+[2+2+2]$, etc., et d'autre part, les femmes en $[3+3]+[3+3]$, etc. Une forme de bimétrie en relation de 2 pour 3 est ainsi superposée à la mélodie chantée, et l'oreille oscille constamment entre plusieurs regroupements métriques possibles, d'autant plus que la pulsation de départ de chacun des groupes de claquements ne coïncide pas avec le début de la mélodie. A tout cela s'ajoute un petit motif de percussion continu, de formule $[2+2+1$ $+2]+[2+1+1+2]$, etc., en double vitesse $(d)$. Bref, un bel

10 Cf. Musik in Afrika, dans les références en fin d'article.

11 Pour audition, cf. Musik in Afrika, cassettes d'accompagnement, ex. 5. 
exemple de polyrythmie et de polymétrie combinées pour nos oreilles occidentales.

Retournons à la première Étude de Ligeti, et voyons comment cette pensée polyrythmique et polymétrique est assimilée par le compositeur. Tel qu'il a été mentionné précédemment, la mélodie de base s'articule en 3 éléments, soit : un premier élément $a$ de 4 mesures en $8 / 8$ (regroupées en $[3+5],[3+5],[5+3],[8])$; puis, un deuxième élément $a^{\prime}$ suivant la même subdivision; et, enfin, un troisième élément $b$ de 6 mesures (regroupées, cette fois, en $[3+5],[3+5],[5+3],[3+5],[5$ $+3],[3+5])$. Cette mélodie est reprise inlassablement tout au long de la pièce, 14 fois en tout, avec cette différence que chaque répétition es $t$ transposée d'un degré vers l'aigu dans l'échelle locrienne (revoir l'exemple 1). On peut considérer les dix répétitions initiales comme une première section $\mathrm{A}$, environ les $3 / 5$ de' 1 'Étude (mes. 1-99). Les trois premières répétitions sont intégrales, mais à partir de la quatrième, Ligeti réalisera une compression graduelle de la structure mélodique : les mesures "perdront" progressivement des pulsations; de 8, au départ, on aboutira à 4 à la dixième répétition (mes. 92). Ce procédé se traduira pour l'auditeur par une impression d'accélération globale, les notes de la mélodie accentuées en octaves apparaissant de plus en plus rapprochées dans le temps. De plus, à partir de la cinquième répétition (mes. 57), le nombre de mesures de chacun des éléments $a, a^{\prime}$ et $b$ se verra lui-même réduit, conduisant à une élimination graduelle des notes accentuées de la mélodie (voir exemple 11).

Un phénomène extrêmement intéressant se produit alors : la distinction entre l'avant-plan (les notes accentuées de la mélodie) et l'arrière-plan (les lignes diatoniques ascendantes) s'abolit au fil des répétitions tronquées! $L^{\prime}$ ordre métrique et rythmique du début cède la place à un contour fluctuant où les uniques points de repère sont les apparitions irrégulières du "motif de tête". Â la onzième répétition (mes. 99), le processus compression-accélération s'étant épuisé, on en arrive à une "cassure" du discours musical; comme c'est très souvent le cas chez Ligeti, le processus s'annihile lui-même, il s'auto-détruit! La mélodie sera ensuite reprise dans sa forme originale pour quatre répétitions ultimes, poursuivant sa course vers l'extrême aigu.

À tout cela s'ajoute, en cours de morceau, un autre niveau de structuration, et c'est par là que cette Étude Désordre prend toute sa dimension polyrythmique (et, jusqu'à un certain point, polymétrique) : au début de la pièce, la main droite (en mode locrien, heptatonique) et la main gauche (réalisant un miroir déformé pentatonique) sont synchronisées, c'est-à-dire que l'articulation rythmico-métrique de l'une 
et l'autre coïncide exactement. Mais dès la cinquième mesure, il se produit un décalage : la quatrième mesure de la main droite se voit amputée d'une pulsation (elle n'a que 7 pulsations), ce qui la fait "sauter" légèrement en avance de la main gauche; et la mesure suivante poursuivra dans le $8 / 8$ original. (Voir, exemple 1, les "amputations" signalées par des crochets [ ]].) Cette "amputation locale" se produira ainsi toutes les quatre mesures : les deux mains seront de plus en plus en décalage, puis se rapprocheront à nouveau pour redevenir synchroniques aprè 32 mesures ( 8 décalages successifs, au rythme de 1 toutes les 4 mesures, pour un cycle complet). Notons toutefois que $l^{\prime}$ ordre des éléments $a, a^{\prime}$ et $b$ fluctuera librement à la main gauche dès la deuxième répétition, renforçant rapidement la polyrythmie naissante. Ligeti suivra cette technique fidèlement, puis, à partir de la quatrième répétition (mes. 43), au moment où la structure globale se compresse, l'idée de déphasage sera développée plus "librement", les deux mains perdant ou regagnant tour à tour des pulsations, cherchant en vain à retrouver leur point de synchronisation.

La première section de l'Étude, soit $\mathrm{A}$ (comme nous l'avons vu : les 10 premières répétitions, jusqu'à la cassure), suggère donc une écoute à plusieurs niveaux. D'une part, à travers les déphasages progressifs émerge lentement une polyrythmie extrêmement riche : de légers déphasages de 1 ou 2 pulsations sont interprétés par l'oreille comme de simples décalages; au-delà de 3 pulsations de déphasage, les structures mélodico-rythmiques des deux mains tendent à être interprétées comme entités indépendantes. D'autre part, au-delà d'un certain taux de compression de la structure mélodique, l'oreille perd les références de l'avant-plan et de l'arrière-plan ainsi que la référence des appuis métriques, et l'attention oscille constamment entre les strates indépendantes superposées aux deux mains; l'auditeur en arrive donc à interpréter la grande densité d“'information" à laquelle il est soumis comme une sorte de "chaos organisé". On comprend maintenant mieux le titre de cette première Étude! En fait, on oscille périodiquement entre une impression d'ordre et de désordre, ou mieux : on glisse imperceptiblement de l'ordre au chaos. ${ }^{12}$

Voyons maintenant la deuxième et dernière section, B. Après la "cassure" où l'on vient de s'arrêter, interviennent 4 nouvelles répétitions de la structure mélodique $a, a^{\prime}, b$, dans sa forme quasi originale : il s'agit

12 Idéalement, le lecteur devrait, à ce stade-ci, réécouter la première section de l'Étude. 
des $11 \mathrm{e}, 12,13 \mathrm{e}$, et $14 \mathrm{e}$ répétitions. La $11 \mathrm{e}$ répétition débute par un canon subtil entre les deux mains, alors que les $12 \mathrm{e}$ et $13 \mathrm{e}$ répétitions (mes. 113 et 127, respectivement) proposent un nouveau type de déphasage : une pulsation sera ajoutée toutes les trois mesures à la main gauche, créant de nouveau "l'autonomie graduelle" des structures mélodico-rythmiques à chacune des mains du pianiste. À partir de la 14e et dernière répétition (mes. 141), la structure mélodico-rythmique de la main gauche se dilate encore plus (les lignes de l'arrière-plan gagnant en importance), jusqu'à se perdre dans le suraigu avec la main droite. Cette section B crée une impression de "pseudo-stabilité" en comparaison avec la section initiale; en fait, elle agit comme à rebours, en nous faisant réévaluer notre perception de toute la section $A$ !

Un dernier point encore mériterait d'être mentionné : il s'agit de l'utilisation simple mais efficace des tessitures du piano. Comme nous l'avons vu, tout au long de la pièce, la main droite se déplace très progressivement du registre moyen au registre suraigu. La main gauche, quant à elle, débute également dans le registre médium, mais elle se dirigera vers le grave jusqu'à l'extrême grave à la fin de la section $\mathrm{A}$, puis se stabilisera dans le registre moyen pendant la section $B$, pour finalement rejoindre la main droite, à la toute fin, dans le suraigu. Nous avons donc une sorte d'ouverture en éventail pour la section A, ce qui permet de suivre les deux strates musicales gagnant progressivement en autonomie; puis, une certaine stabilisation pour la section $\mathrm{B}$, tout à fait conforme au caractère de la musique. Fait notable, dans la section $B$, Ligeti remplace les octaves correspondant aux notes accentuées par des accords de 2,3 , puis 4 sons, de façon à donner aux accentuations plus de relief dans l'aigu et le suraigu.

$\mathrm{Si}$, à cette phase terminale de l'analyse, on réécoute l'Étude Désordre dans sa totalité, on s'aperçoit que la pièce est le lieu d'une symbiose très particulière : Ligeti semble avoir "syncrétisé" la pensée en regroupements métriques asymétriques typique de plusieurs musiques des Balkans, de même que des traditions arabes syrio-égyptiennes et irakiennes, avec la pensée polyrythmique des musiques africaines subsahariennes. Le tout est formalisé en un processus de fluctations subtilement organisé, et, en cela, très caractéristique de toute la musique d'un compositeur passé maître, depuis trente ans, dans la combinatoire de mécaniques de haute précision!

\section{Etude 2 : CORDES VIDES}

Cordes vides est un Andantino en teintes toutes "impressionnistes", contrastant avec la première Étude (voir exemple 12). 
La structure harmonique est réalisée par des superpositions de quintes s'étalant sur toute l'étendue du clavier et faisant ainsi référence aux cordes à vide des instruments à cordes. L'impression générale en est une de "flottement" : les quintes se déploient en phrases asymétriques, formant des mélodies de 4,5,6,7 sons, jusqu'à 13, en formules tantôt ascendantes, tantôt descendantes, tantôt revenant sur elles-mêmes ou progressant chromatiquement. Les phrases étant de longueur inégale à chacune des deux mains, l'impression de flottement harmonique se voit renforcée par un "flottement métrique"; en fait, l'oreille ne peut que s'attacher à suivre de grandes hémioles, des patterns de longueur fluctuante se déplaçant dans l'espace et dans le temps. Au début de $l^{\prime}$ Étude, les deux mains évoluent suivant une pulsation commune. Mais bientôt, le mouvement s'agitera et chacune des mains adoptera sa vitesse propre, progressant de plus en plus vers des proportions de 2 pour 3. L'écriture se fait alors plus virtuose, une même main "balayant" rapidement plusieurs tessitures du piano - technique chère à Liszt ou à Chopin... Le "balayage" rapide miroitera, vers la fin, de la main droite à la main gauche, pour bientôt se perdre dans les résonances de l'extrême grave. Ici, l'habitus de la musique de piano romantique est comme sous-entendu, en arrière-plan, mais le caractère a quelque chose de vaguement nostalgique, de contenu; musique fugace, fluide, qui n'est pas sans rappeler le climat du début de la 1le Etude pour piano de Debussy, Pour les arpèges composés (voir exemple 13).

\section{Etude 3 : TOUCHES BLOQUEES}

Le titre de cette Étude réfère directement à la technique de jeu employée pour la première fois par Ligeti dans la deuxième des Trois pièces pour deux pianos : Selbsportrait mit Reich und Riley (und Chopin ist auch dabei). ${ }^{13}$ Ligeti renvoie lui-même, dans la partition, à un article de Henning Siedentopf datant de 1973 et décrivant cette nouvelle technique : une des deux mains enfonce (silencieusement) et tient certaines touches du piano notées par un petit losange dans la partition; l'autre main joue les motifs notés normalement, en valeurs brèves continues. Quand, par exemple, la main droite "joue" une touche déjà enfoncée par la main gauche, aucun son n'est produit et une courte pause vient interrompre le flot continu. Cette technique permet d'exécuter de manière extrêmement rapide des rythmes irréguliers complexes sans que le pianiste ait à compter la durée exacte de chacune des notes. Ligeti utilise cet idiome pianistique pour créer une fluctuation très rapide de

13 Autoportrait avec Reich et Riley (Chopin est aussi "de la partie"). 
syncopes virtuelles, un tapis métrique en mouvance constante, jonglant avec des regroupements irréguliers de pulsations brèves. Le sentiment de "pseudo-régularité" métrique du début ne sert qu'à mieux nous dérouter par la suite. (Voir exemple 14.)

La texture de l'Etude est en grande partie monodique mais, comme le court exemple 14 le montre déjà, la mélodie principale se dédouble fréquemment en 2 ou même 3 lignes "inhérentes", tout à fait dans l'esprit des patterns inhérents de la musique africaine dont il a été question précédemment. ${ }^{14}$ Les lignes mélodiques évoluent en une forme de plurimodalité fluctuante, $l^{\prime}$ une des structures mélodiques privilégiées consistant un pentacorde lydien, "ancré" au départ sur une tonique si, auquel vient s'ajouter par moments une terminaison phrygienne (voir exemple 15).

La couleur modale, bien que constamment variée, transposée ou brouillée par le chromatisme, fait directement allusion à la musique folklorique roumaine, dans laquelle il est typique de combiner ou de superposer plusieurs modes. Un exemple bien connu est la combinaison fréquemment utilisée par Bartók et qu'il a dénommée mode acoustique: un pentacorde lydien (par exemple : do, ré, mi, fa dièse, sol) est suivi d'une terminaison myxolydienne (la, si bémol, do). La parenté avec le mode de départ employé ici par Ligeti est frappante (voir exemple 16).

Peut-être pourrait-on voir dans Touches bloquées une forme "éclatée" de pluri et de polymodalité, sorte d'extrapolation de la pensée modale des traditions balkaniques ... Et, justement, par rapport à la pensée modale, il serait certainement intéressant de jeter un coup d'oeil rétrospectif sur les contours mélodiques dans la production de Ligeti...

\section{Etude 4 : FANFARES}

La quatrième Étude est une sorte de reprise nouvellement développée $\mathrm{du}$ deuxième mouvement (Vivacissimo molto ritmico) du Trio pour violon, cor et piano (1982). Il s'agit d'une musique très directe, avec un ostinato diatonique perpétuel comme trame de fond sonore - des mesures de 8 pulsations rapides, regroupées en $[3+2+3]$. (Voir exemple 17.)

On peut y entendre à loisir une allusion à la musique des Balkans, en particulier au fameux Aksak dont il a été question au sujet de la

14 Voir, à titre de comparaison, les 2 patterns inhérents de l'exemple 2. 
première Étude, tout aussi bien qu'à la Salsa des Caraïbes ou encore, à la polyrythmie typique de la Rumba cubaine et de la Samba brésilienne, avec les accentuations asymétriques des claves et des timbales en rythmes dits tresillo (3 pulsations asymétriques) ou cinquillo (5 pulsations asymétriques, réparties sur deux mesures). Quelques illustrations schématisées du tresillo et du cinquillo sont montrées à l'exemple 18, mais le lecteur-auditeur pourra aussi consulter les nombreuses anthologies de sambas brésiliennes ou de rumbas cubaines qui existent sur le marché. 15

Ligeti part de ce principe de combinatoire en articulations asymétriques pour réaliser une polymétrie en perpétuelle variation : au-dessus de l'ostinato se dessinent des lignes mélodiques irrégulières, toutes parentes mais toutes légèrement différentes dans leur contour, leur durée et leur articulation rythmique. L'oreille tend, à chaque détour, à regrouper les pulsations en "mètres cohérents", mais le taux de variation est tel que l'on "perd pied", littéralement; on pourrait certainement parler ici d'une application particulièrement originale du principe de l'hémiole à plusieurs niveaux de structure simultanés. Au plan harmonique, l'auditeur se voit tout aussi dérouté : l'ostinato lui-même emprunte son premier tétracorde à la tonalité de do majeur, mais son deuxième "glisse" légèrement vers si majeur (voir exemple 17, mes. 1, main gauche). La mélodie, le plus souvent harmonisée à deux voix ou se dédoublant en arpèges brisés, favorise les consonances de tierces et quintes, avec leurs renversements, mais dans un contexte "hors tonal" : une série particulière de 12 sons est traitée, comme c'est le cas dans le Trio pour violon, cor et piano, de façon à générer constamment de nouveaux regroupements de constellations de deux sons. Le titre Fanfares fait allusion aux sonneries de cuivres stylisées qui sous-tendent

15 Pour la diffusion radiophonique originale du présent texte, $j$ 'avais choisi un exemple de salsa. La salsa, née aux États-Unis dans les années 60 et réunissant des groupes de vedettes ("all-star-bands") composé, en majeure partie, d'exilés cubains, combine - comme dans un creuset - plusieurs des éléments essentiels de la rumba ou du cha-cha cubains, du meringue mexicain et du reggae jamaïcain. Le terme fut vraisemblablement employé pour la première fois au début des années 60 par Jerry Masucci, pour sa série d'enregistrements sur étiquette Fania. L'exemple choisi était la salsa "Azucar", avec son articulation asymétrique de la ligne de basse en tresillo, typique de la rumba, ses lignes mélodiques en moultes variantes "syncopées" s'étalant sur plusieurs mesures et les traits en hémioles typiques des cuivres. Cf. Jerry Masucci et son ensemble, Salsa Greats, disques Fania, Sono-disc (SD 15). 
les contours mélodiques. On trouvera, à l'exemple 19, une sonnerie typique de cuivres, basée sur les harmoniques naturels, et, à l'exemple 20 , quelques illustrations tirées de la partition de Ligeti.

\section{Etude 5 : ARC-EN-CIEL}

Dans toute sa fine nostalgie, la cinquième Étude (voir exemple 21) prend les allures d'une musique filtrant d'un "salon", ou mieux, d'une "alcôve" imaginaire où se profilent les ombres du Schumann sublime des Phantasiestücke (cf. entre autres, Des Abends, première pièce de ce recueil dont le début est montré à l'exemple 22), les arabesques impressionnistes d'un Fauré ou d'un Duparc, ou encore, quelque écho d'un ballade d'Errol Garner ou de Bill Evans (voir, exemple 23, un aperçu de l'introduction à Nirvana, de Herbie Mann). ${ }^{16}$

Dans Arc-en-ciel, la couleur harmonique repose principalement sur des progressions d'accords majeurs ou mineurs, avec septièmes et neuvièmes ajoutées, sonorités fluides s'il en est... (Le cycle harmonique du début de la pièce est montré à l'exemple 24). Ces progressions d'accords se développeront tantôt de manière synchrone, aux deux mains, mais tantôt égalerent de façon asynchrone générant ainsi de nouvelles constellations harmoniques qui tendent à une forme de bitonalité épisodique. (Voir, exemple 21, la bitonalité émergeant graduellement des mes. 8 à 11.)

L'écriture développe la pensée en hémiole, typique des œuvres de Chopin et de Schumann; qu'il suffise de se rappeler le début de Des Abends (exemple 22), où les 6 pulsations de chaque mesure sont articulées en 3 groupes de 2 notes à la main droite et 2 groupes de 3 notes à la main gauche, créant le doux flottement agogique qui fait tout le charme du morceau. Dans Arc-en-ciel, chaque mesure de 12 pulsations régulières est divisée en 3 motifs de 4 notes à la main droite et 2 motifs de 6 notes à la main gauche, chaque main créant en outre 2 voix "virtuelles" de par le jeu arpégé (voir, exemple 21, les mes. 1-2).

L'articulation de la main droite aura tendance à fluctuer au cours de la pièce, des regroupements en 2 et 3 pulsations apparaissant à l'occasion; de plus, la régularité de la pulsation variera de manière subtile et expressive par la production de "déphasages locaux", chaque main

16 Pour audition, cf. Atlantic Jazz Collection, volume "piano", Atlantic (781 707-1). Bill Evans, piano, Herbie Mann, flûte, Chuck Israels, basse, Paul Motian, batterie. Enregistré au début des années 60 . 
évoluant par endroits à sa propre vitesse. Le discours "s'enflera" périodiquement, créant des montées de tension, des "élans lyriques" se résolvant tour à tour sur des reprises, ou plutôt, de "fausses" reprises $\mathrm{du}$ thème toujours modifiées. ${ }^{17}$ Le tout se dissout sans conclusion véritable dans une "évaporation" vers l'extrême aigu..., comme si le prisme décomposant la lumière blanche en arc-en-ciel se voilait peu à peu....

\section{Étude 6 : AUTOMNE À VARSOVIE}

Comme la première Étude - Désordre -, Automne à Varsovie est une pièce de haute virtuosité, mais d'une virtuosité toute particulière : Ligeti combine une conception élargie de la technique de l'hémiole de la musique romantique avec la pensée en patterns additifs de la musique africaine, pour en arriver à donner l'illusion de plusieurs strates de vitesses superposées et ce, exécutées par un seul interprète. Le pianiste joue, en arrière-plan, une succession rapide et régulière de 16 pulsations par mesure à 4/4; au départ, il s'agit de regroupements de 4 pulsations en octaves brisées, mais ce pattern se verra constamment modifié en cours de morceau. Le matériau mélodique - montré à l'exemple 25 est très près de celui employé dans le quatrième mouvement du Trio pour violon, cor et piano ou, encore, dans le deuxième mouvement du Concerto pour piano (1986). Il s'agit d'une mélodie descendante, mi-chromatique, mi-diatonique, d'un "Lamento" à vague saveur modale, suggérant, entre autres, le Bocet roumain - ce chant traditionnel des pleureuses sur la dépouille d'un défunt -, ou rappelant encore les complaintes du Cante Jondo d'Andalousie. Voir, exemple 26, une transcription de Cintecul bradului (Chant du sapin), de Transylvanie : un chant funéraire roumain, en mode phrygien, à l'intention des jeunes défunts morts célibataires. Ce type de motif descendant a été fort souvent utilisé, également, dans la tradition occidentale comme thème de passacaille. Que l'on se souvienne simplement du Moro Lasso de Gesualdo ou, encore, de la "Lamentation de Didon" dans l'opéra Didon et Énée de Purcell. (Å ce dernier titre, voir exemple 27).

Inévitablement, et cela est le cas pour un très grand nombre de cultures musicales, l'impression dégagée en est une de douleur ou de résignation à l'inéluctable. Chez Ligeti, ce motif "Lamento" sert de thème à

17 Par exemple : mes. 13, 3e temps du 3/4; mes. 16, ler temps; mes. 17, 2e temps du 3/4; mes. 20, ler temps avec anacrouse. 
l'élaboration d'un mouvement fugué complexe, où les 4 voix viennent constamment s'entremêler à l'ostinato de l'arrière-plan. Il y a ainsi des endroits dans la pièce où la main droite accentue chaque cinquième pulsation pendant que la main gauche, elle, accentue toutes les trois pulsations (voir exemple 28).

Ces deux chaînes de notes accentuées se combinent dans notre perception en super-signaux et se transforment en deux mélodies qui se déplacent à des vitesses différentes, l'accentuation faite à toutes les 5 notes produisant un tempo plus lent et celle faite à toutes les 3 notes, un tempo plus rapide. Si la relation de 5 à 3 constitue une proportion arithmétique simple, elle est tout de même trop complexe pour notre perception : nous ne comptons pas des pulsations; ce que nous ressentons est plutôt une impression qualitative de tempi superposés. Le pianiste non plus ne "compte" pas : il accentue les notes selon les indications de la partition, il sent à travers ses doigts des patterns de contractions musculaires étalés dans le temps, mais ce qu'il entend est autre chose; il entend une superposition de tempi, telle qu'il n'aurait pu la réaliser consciemment.

Automne à Varsovie comporte aussi des moments qui combinent à la fois 3 et même 4 tempi différents; à ces occasions, les séries de notes accentuées ne sont alors plus seulement réparties entre les deux mains, mais bien entre les doigts $d^{\prime}$ une même main (voir exemple 29).

Ligeti a dû, ici, imaginer une musique se formant à partir des limitations anatomiques inhérentes aux dix doigts sur les touches d'un clavier. En cela, et selon ses propres dires, il s'est référé à Chopin, pour lui, l'idéal de ce point de vue : chez Chopin, aucune trace $d$ une quelconque contrainte exercée sur l'imagination et le contenu poétique par les données physiques du jeu pianistique; bien au contraire, la pensée créatrice du compositeur est comme inconsciemment pré-façonnée, stimulée par les prémisses anatomiques et techniques. En fait, la disparité entre les patterns des contractions musculaires et l'image sonore produite (les patterns auditifs résultants) est une des sources de pur plaisir physique du jeu pianistique, et cela vaut tout autant pour les hémioles plus simples de Chopin que pour les hémioles généralisées de Ligeti. Â titre de référence, le début de l'Étude op. 10 no 10, en la bémol, de Chopin est d'un grand intérêt (voir exemple 30) : la main gauche réalise un motif d'accompagnement articulé en 2 fois 3 pulsations, pendant que la mélodie, à la main droite, semble se déplacer à une vitesse plus grande de par son articulation en $2+2+2$. 


\section{Pour conclure}

Il faut répéter que, dans la musique de Ligeti se perpétuent et se revitalisent plusieurs des éléments les plus riches de la tradition musicale européenne, le syncrétisme personnel du compositeur se situant au carrefour d'un grand nombre de traditions qui débordent largement les cadres de la musique de concert occidentale. Par le biais de maintes transplantations nerveuses, transfusions sanguines et greffes in vivo, Ligeti crée un organisme musical nouveau, viable, refondant les référents culturels et les assumant dans leur potentiel référentiel propre. C'est là un art des plus subtils, des plus sensibles et imaginatifs, et il ne fait aucun doute que les Six Études pour piano, de par leur fraîcheur et leur classicisme raffiné, constituent un apport majeur au répertoire. 


\section{RÉFERENCES}

KUBIK, Gerhard.

1983 : "Kognitive Grundlagen afrikanischer Musik", Musik in Afrika. Berlin : Staatliche Museen Preussischer Kulturbesitz, Berlin Museum für Völkerkunde.

\section{SALAH EL MAHDI}

1972 : La Musique arabe. Paris : A. Leduc.

SCHENBERG, Arnold.

1967 : Fundamentals of Musical Composition, édité par Gerald Strang, avec la collaboration de Leonard Stein. New York : St. Martin's Press.

1975 : Style and Idea, éditée par Leonard Stein. London : Faber and Faber.

SIMON Artur et als.

1983 : Musik in Afrika (recueil d'articles avec cassettes 
Ex. $1: D E ́ S O R D R E$ (1ère des 6 Études pour piano de György Ligeti)
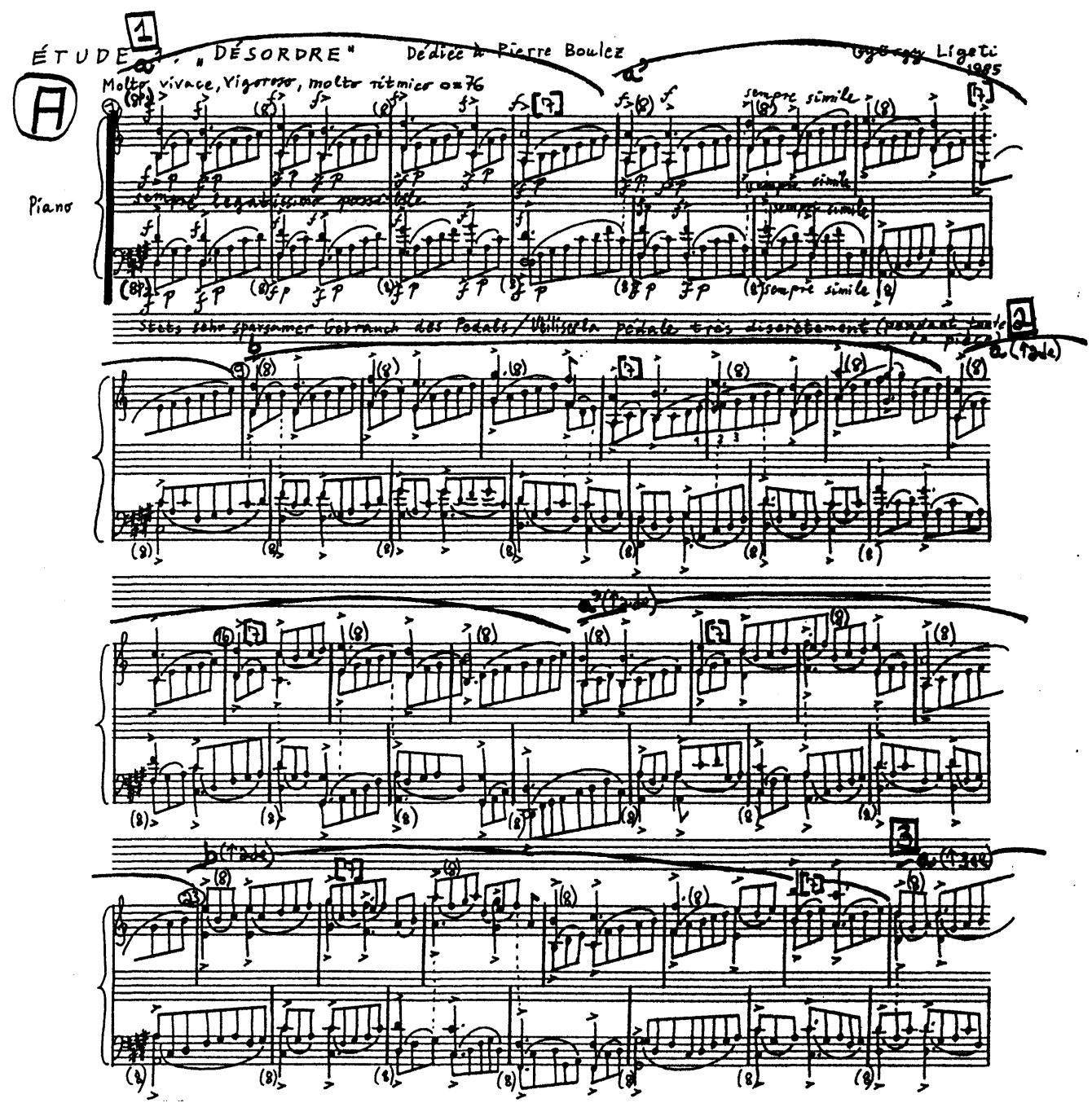

$\bigotimes_{a, a, b}(\uparrow)$ Répétitions de la mélodie avec ses transpositions

$(x),[x]$ nombre de pulsations par mesure

By permission of the Ligeti Publisher, B. Schott's Söhne Mainz. 


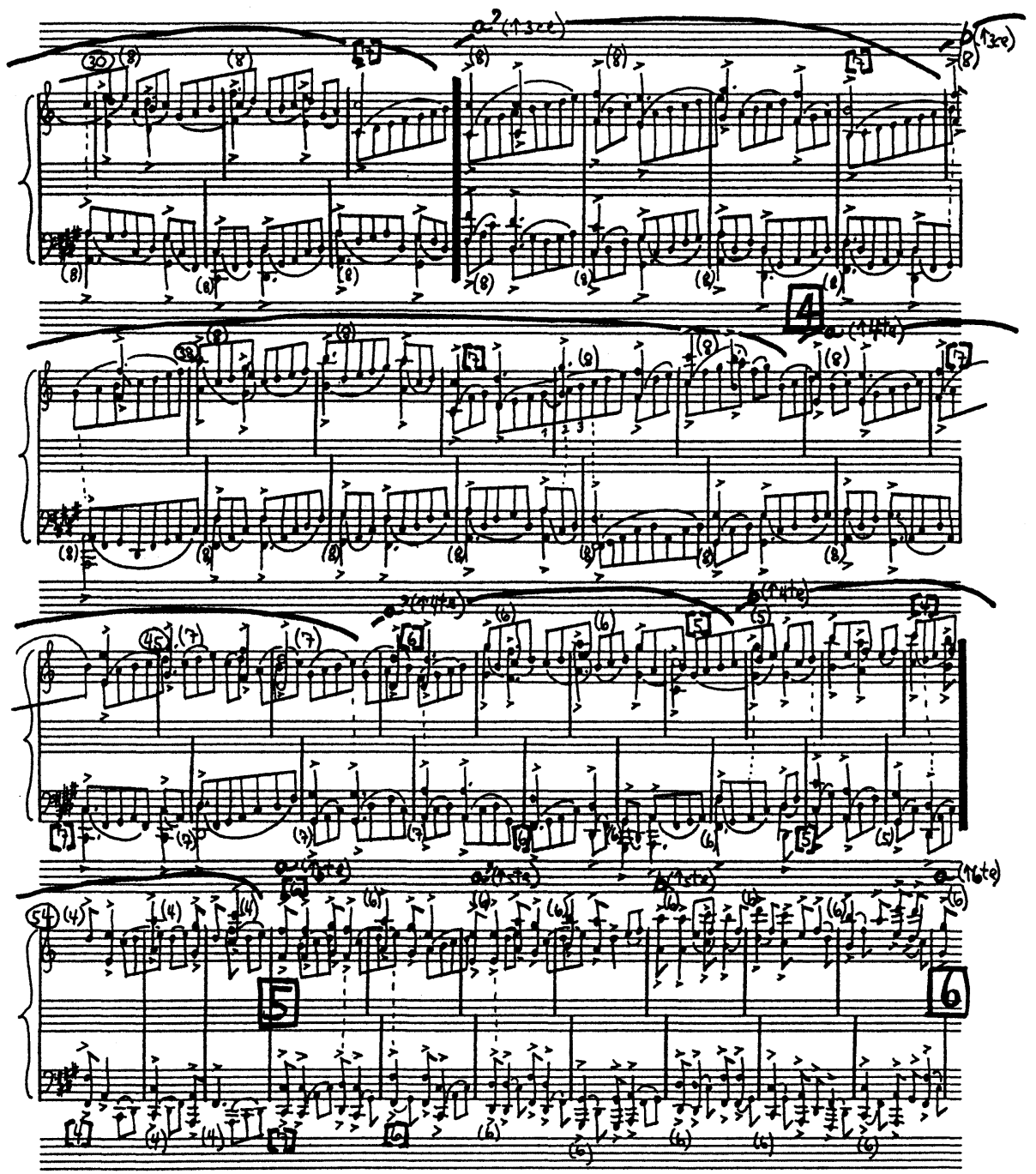



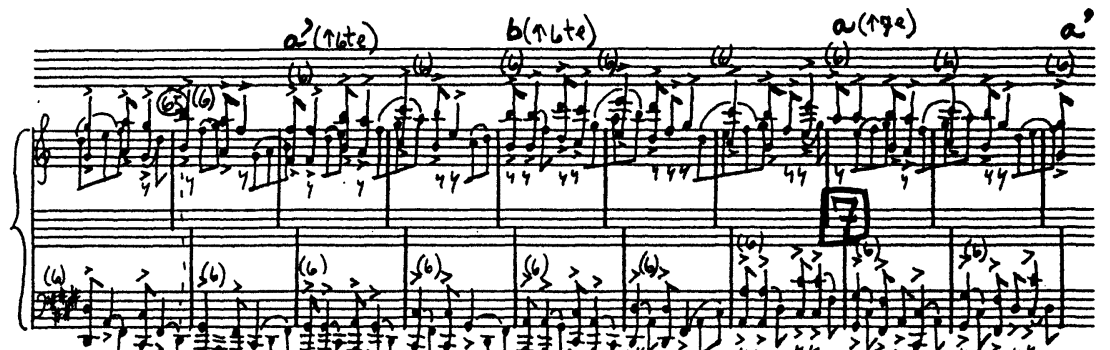

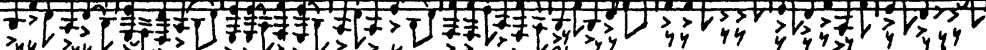

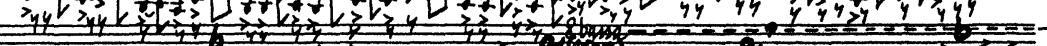

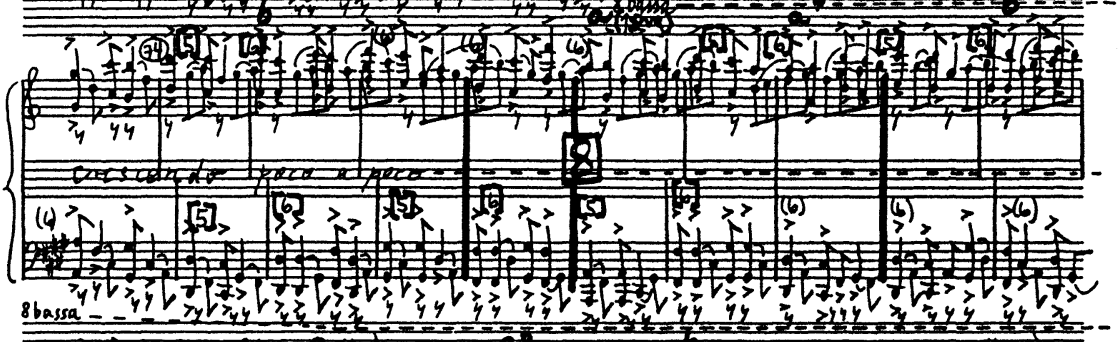

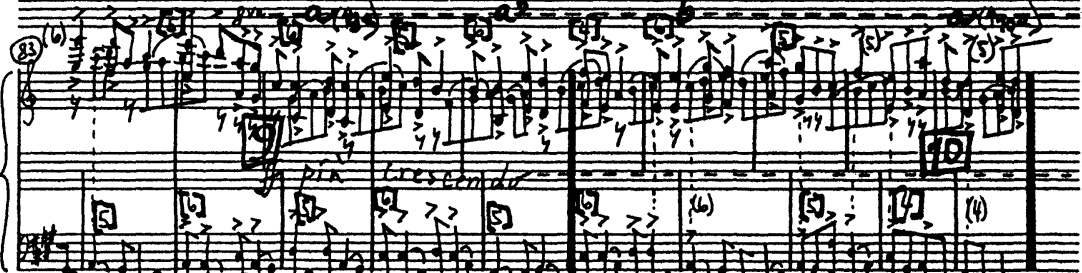

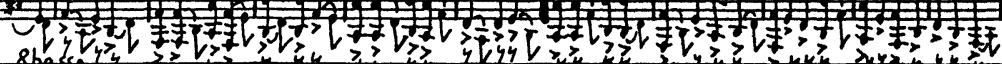

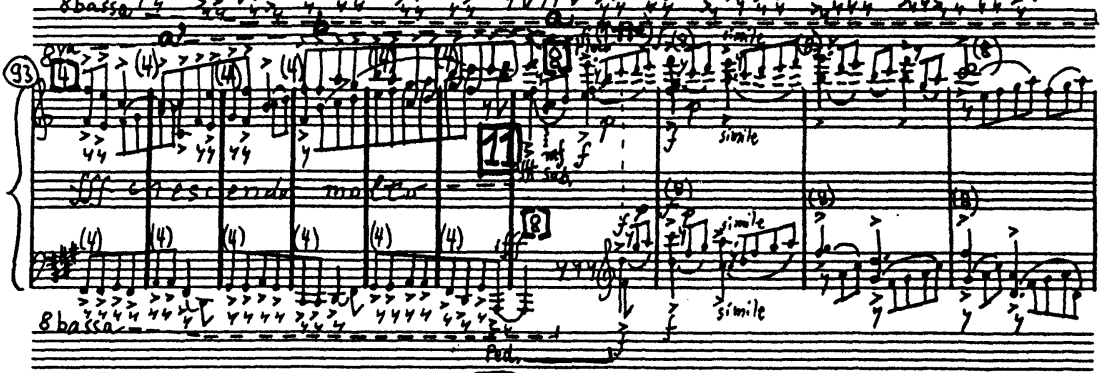

(B) 

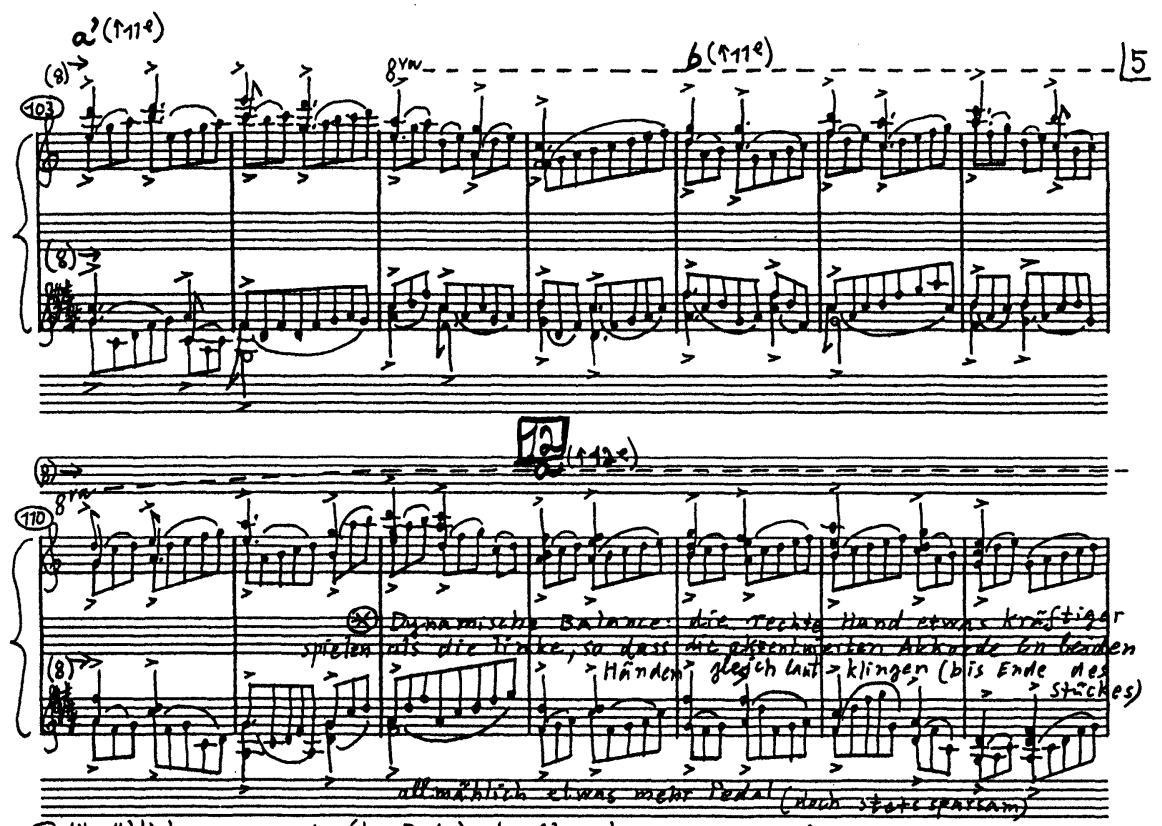

Allmähliches crescendo (bis Ende): die Akzente werden all mählich ff a dann fffe

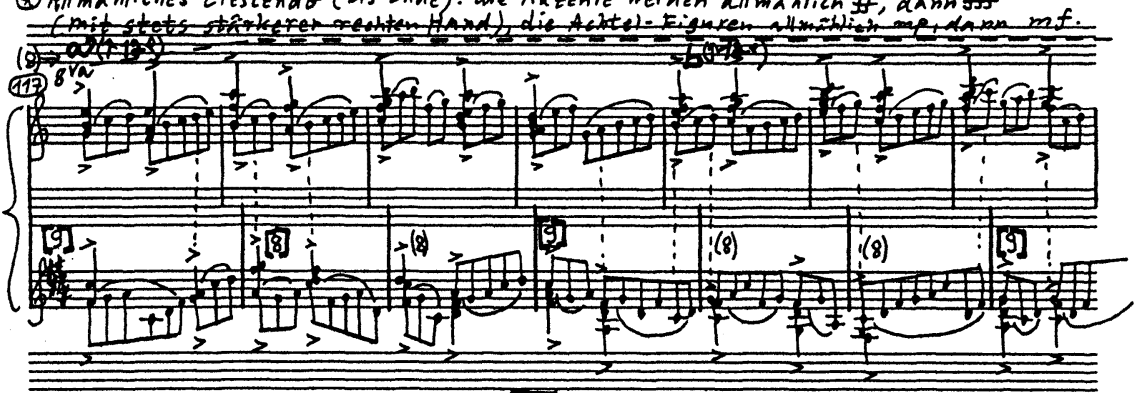

13

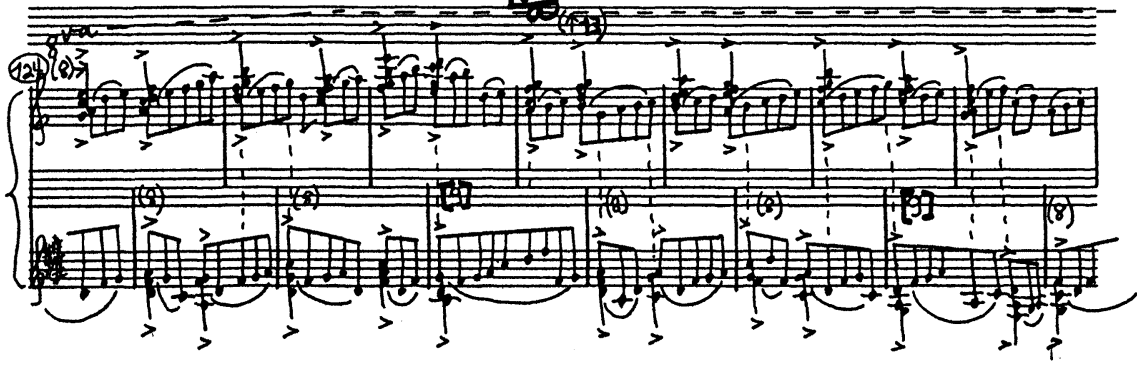



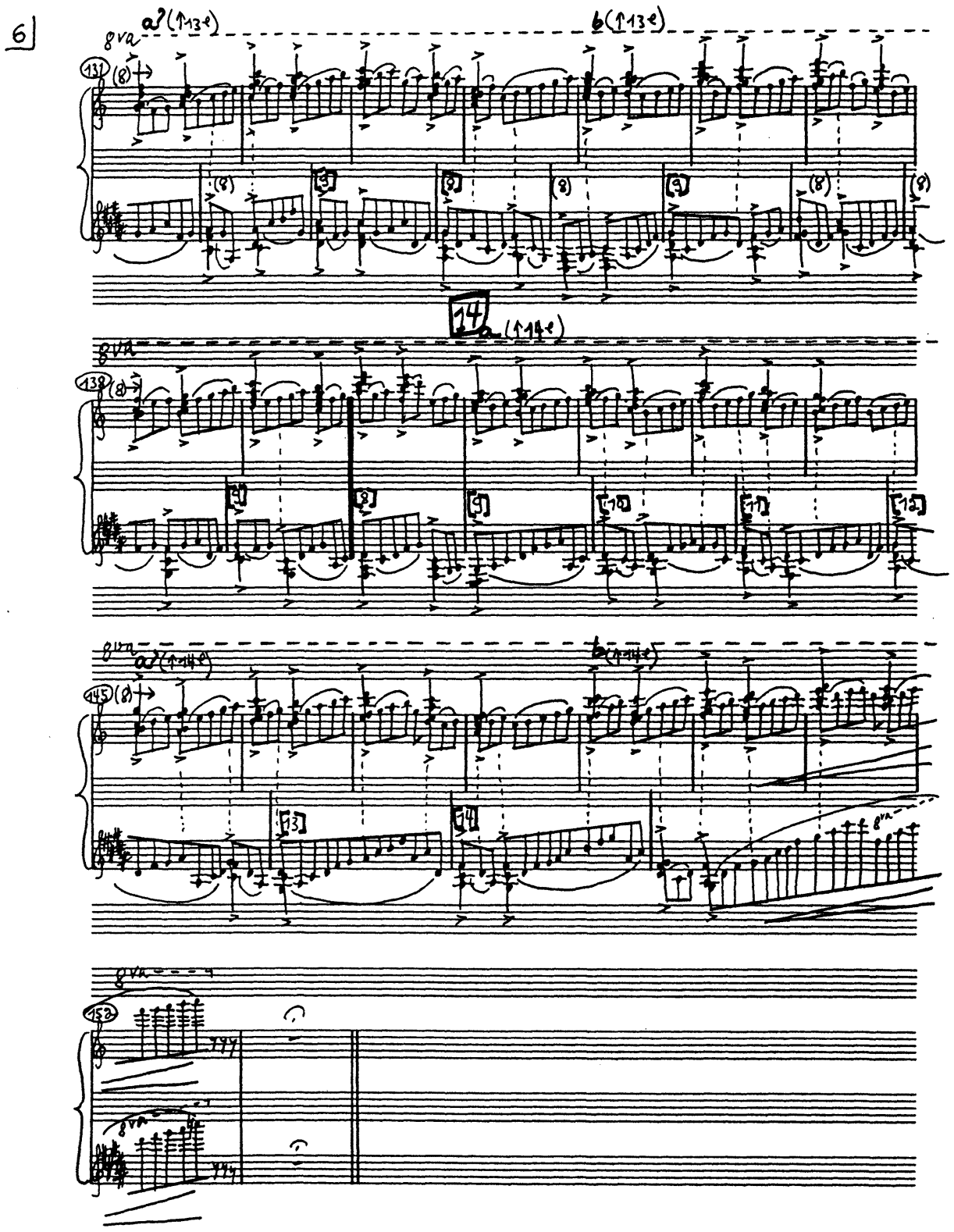

By permission of the Ligeti Publisher, B. Schott's Söhne Mainz. 


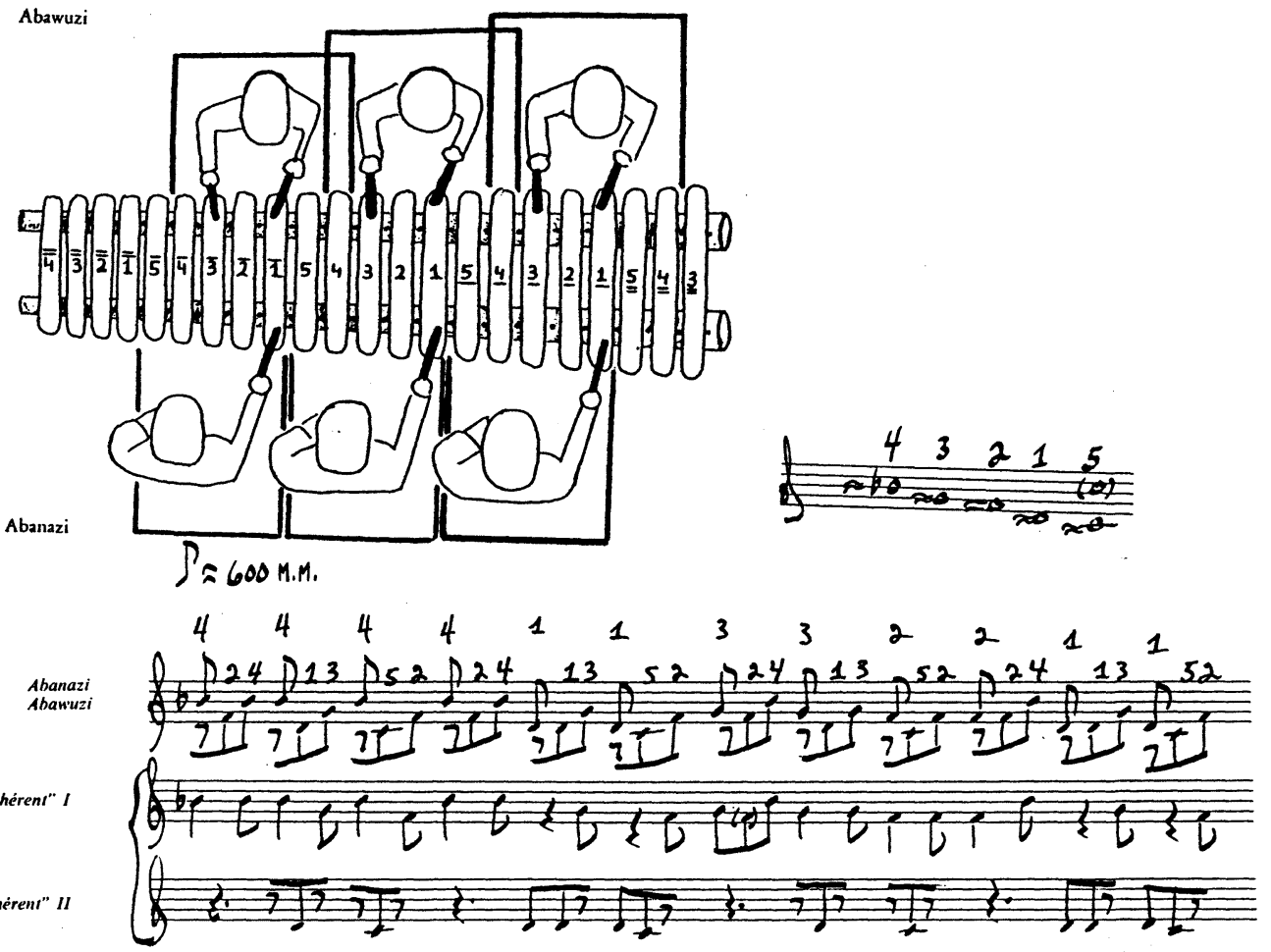

Ex. 2 : Ab'e mbuga basengejja, musique AKADINDA des musiciens de Salama (Ouganda). 
Ex. 3 : "Projection" de l'échelle heptatonique (main droite) sur l'échelle pentatonique (main gauche). Cf. Désordre, mes. 1-8.

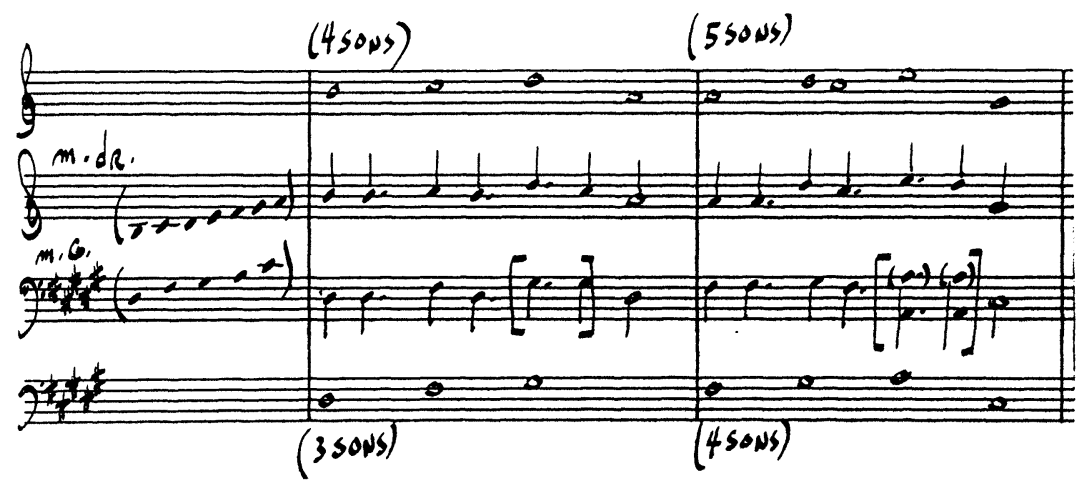

Ex. 4 : Allegro barbaro de Bartók, "motif de tête" (mes. 1-19)
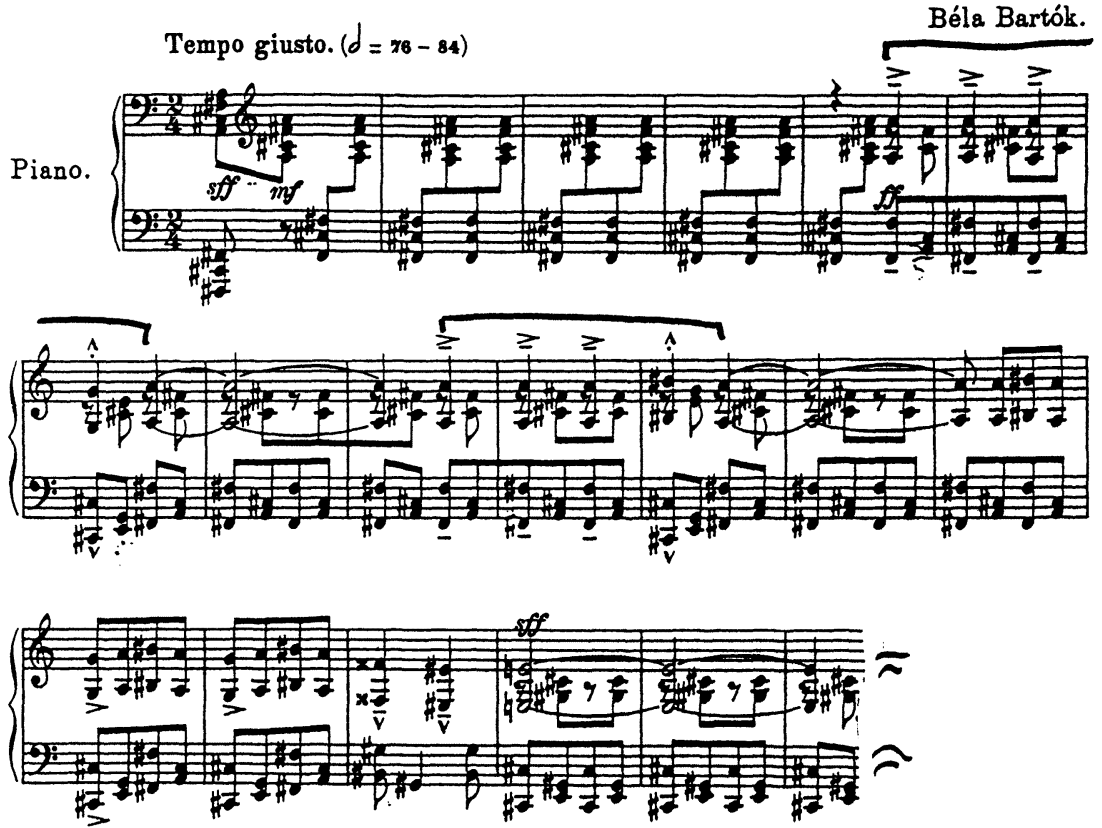
Ex. 5 : Sonate pour deux pianos et percussion de Bartók, "motif de tête" (mes. 33-40).
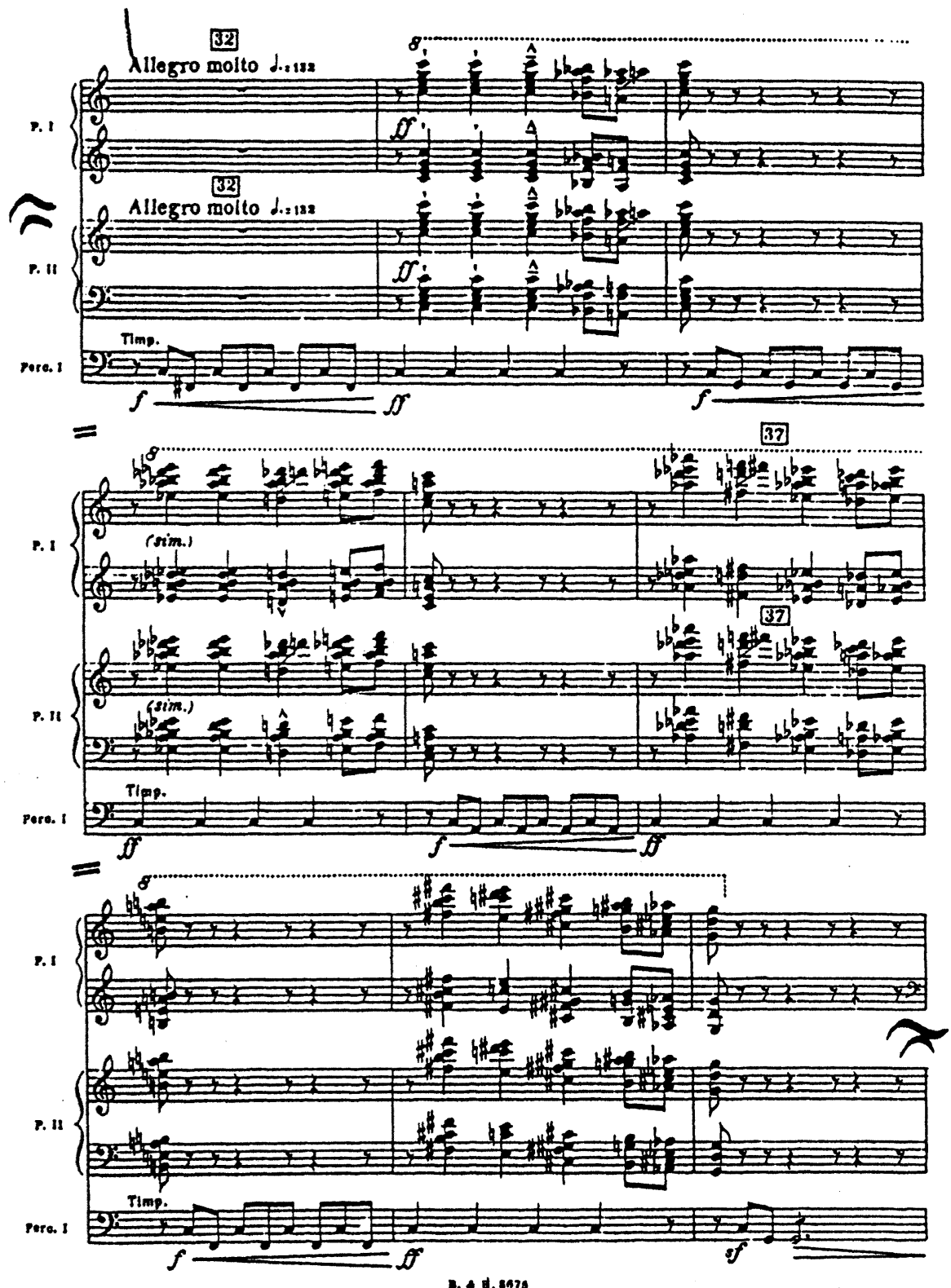
Ex. 6 : Désordre, mélodie de base a, $\mathrm{a}^{\prime}, \mathrm{b}$

(remplissage graduel de l'espace modal heptatonique)

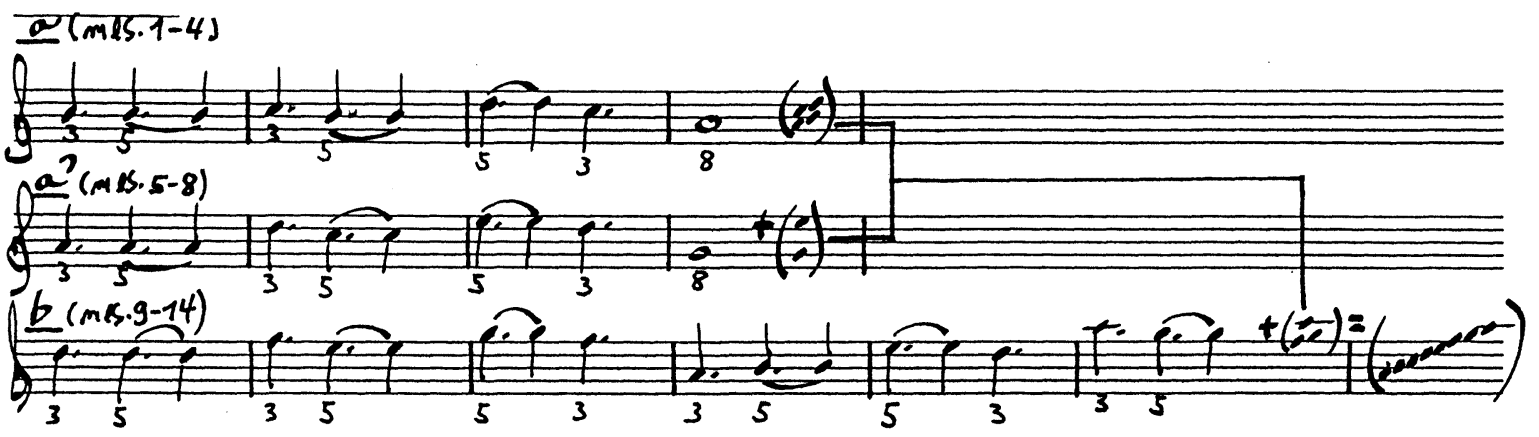

Ex. 7 : Sonate op. 2 no. 3 de Beethoven, thème principal en SENTENCE.

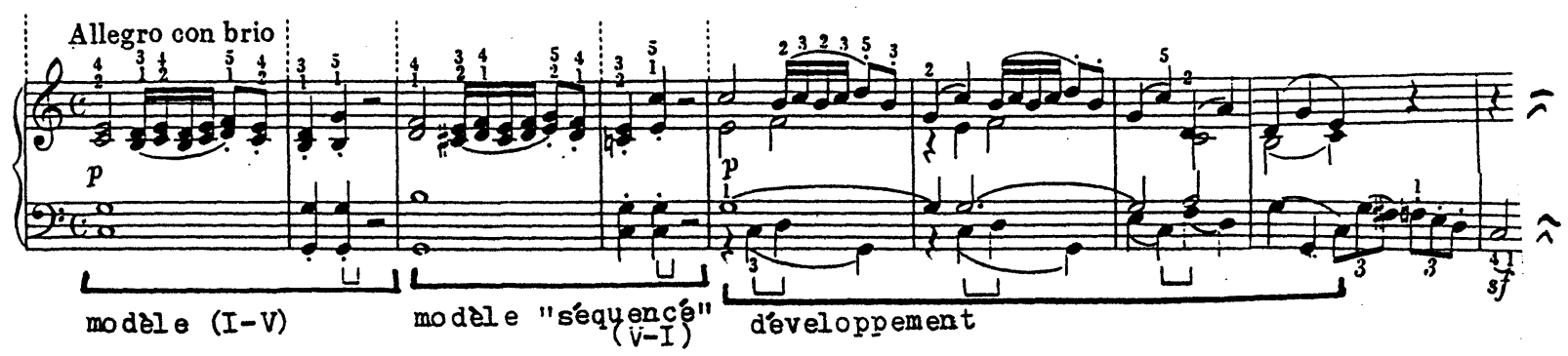


\&
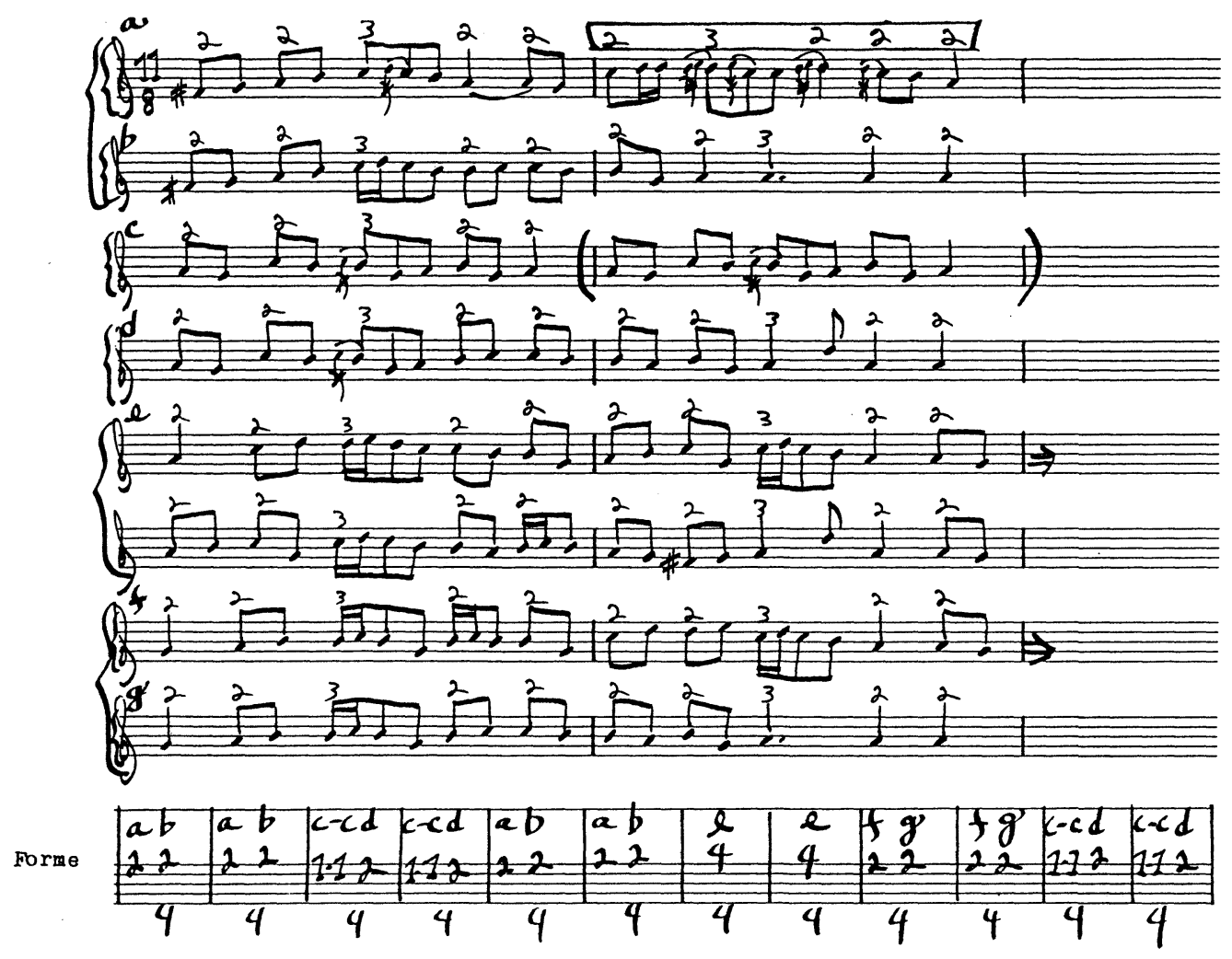

Ex. 8 : Danse bulgare Gankino Horo (1ère partie) structure mélodique, rythmique, schéma formel. 
Ex. 9 : Scherzo du Quatuor à cordes no 5 de Bartók (mes. 1-10), articulation $4+2+3$.

Alla bulgarese, (vivace, d dd...4(i)
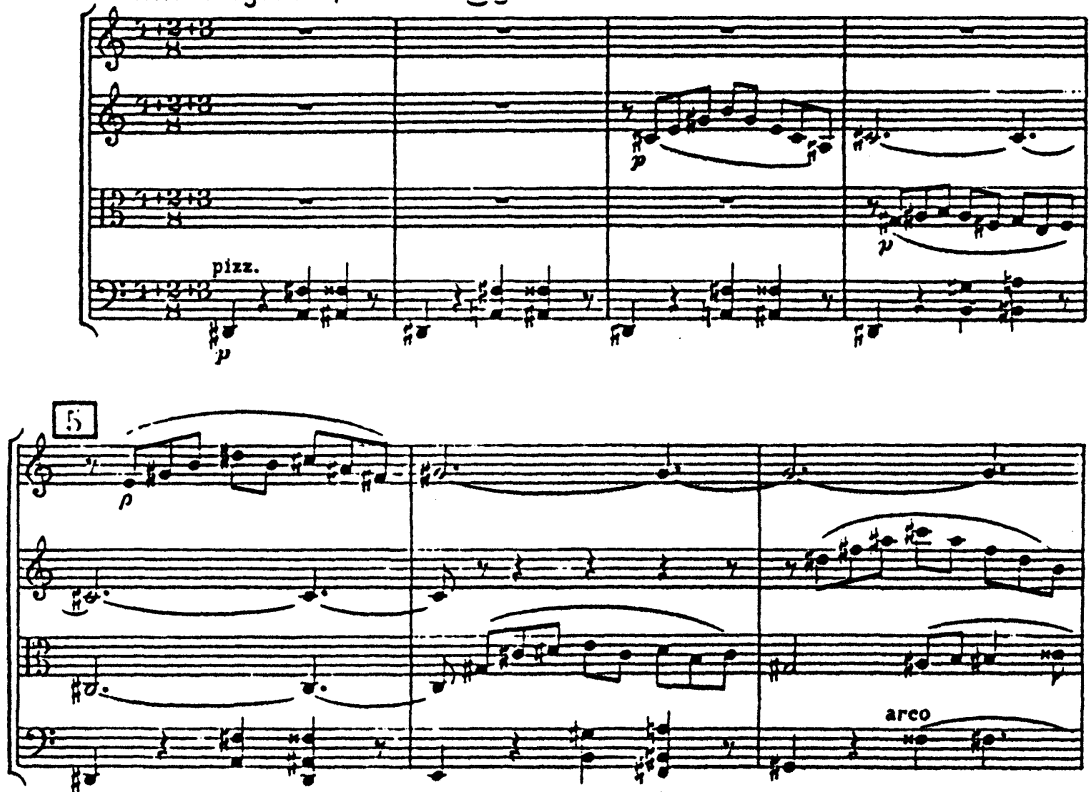

$=$

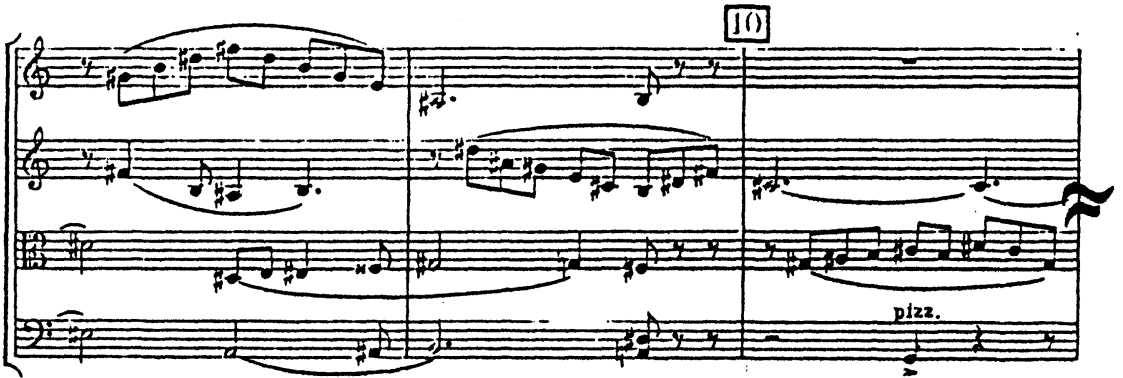




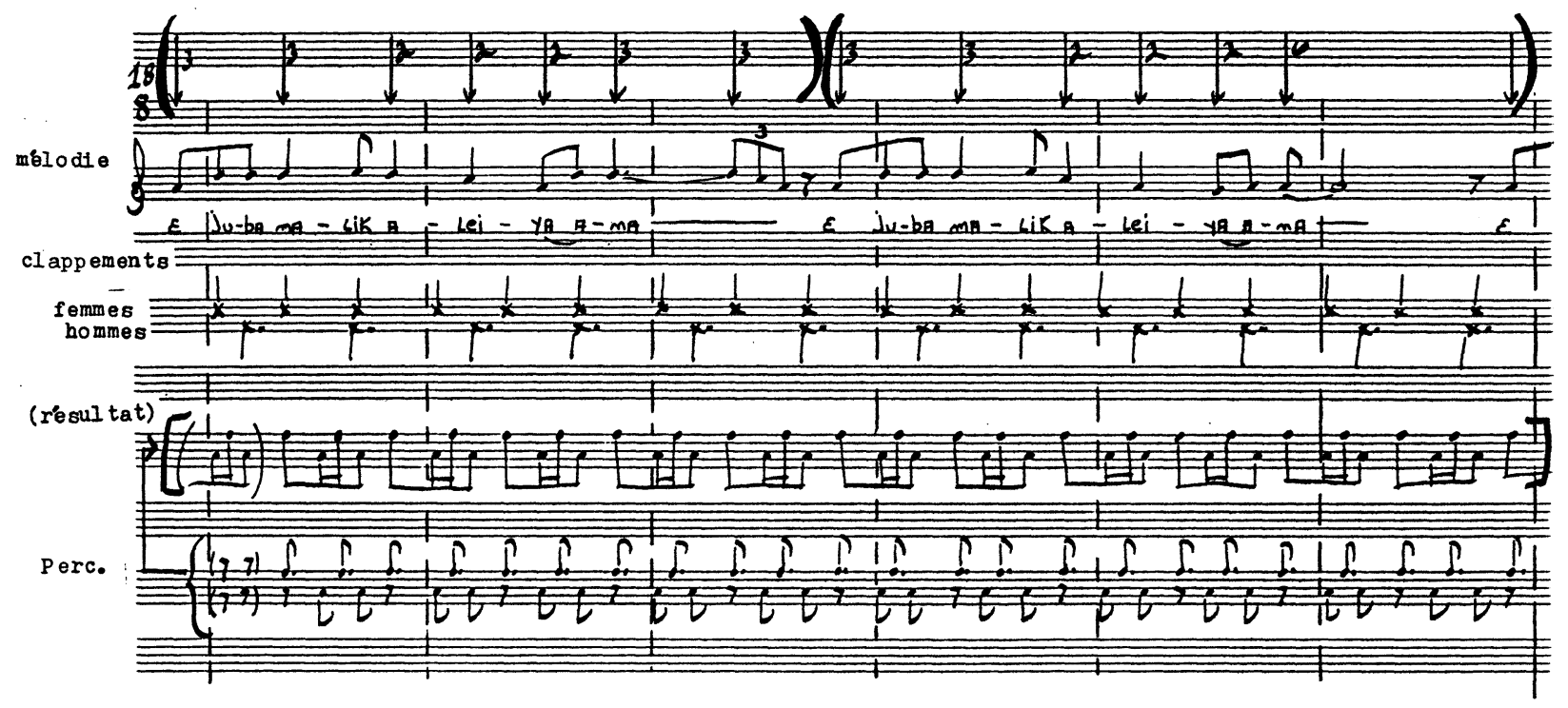

Ex. 10 : E Juba malik aleya ana, Chant de Geigar, Soudan. 
Ex. 11 : Désordre, schéma sommaire de la compression de la mélodie (main droite).

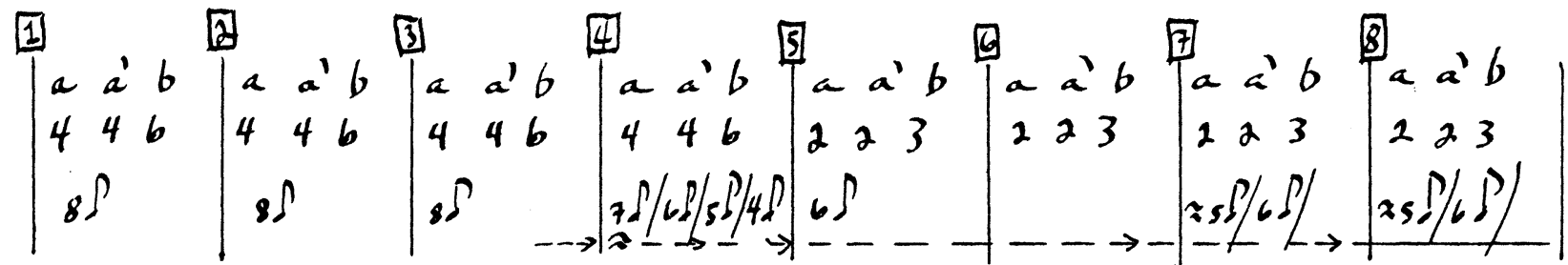

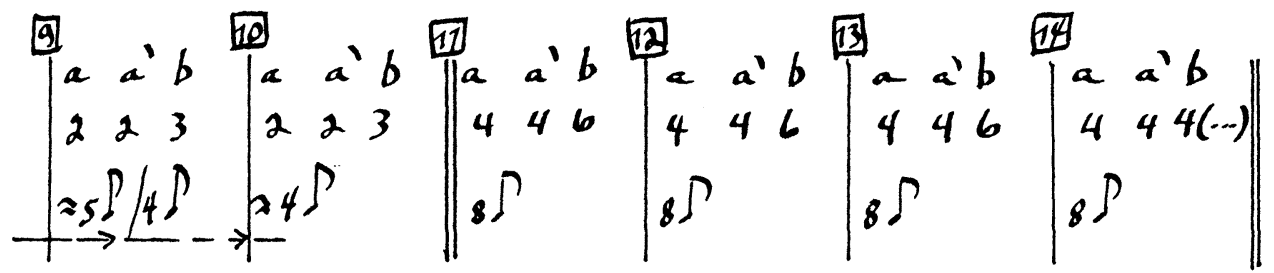

$$
\text { ( } A \text { = Répétitions de la mélodie, voir partition Ex. 1) }
$$

8 
Ex. 12 : Cordes vides, de Ligeti (1ère page)

ÉTUDE 2: "CORDES VIDES" Dédiée à Pierre Boulez

Andantine con mote, melto tenere $S=120$ Gö̈rgy Ligeti
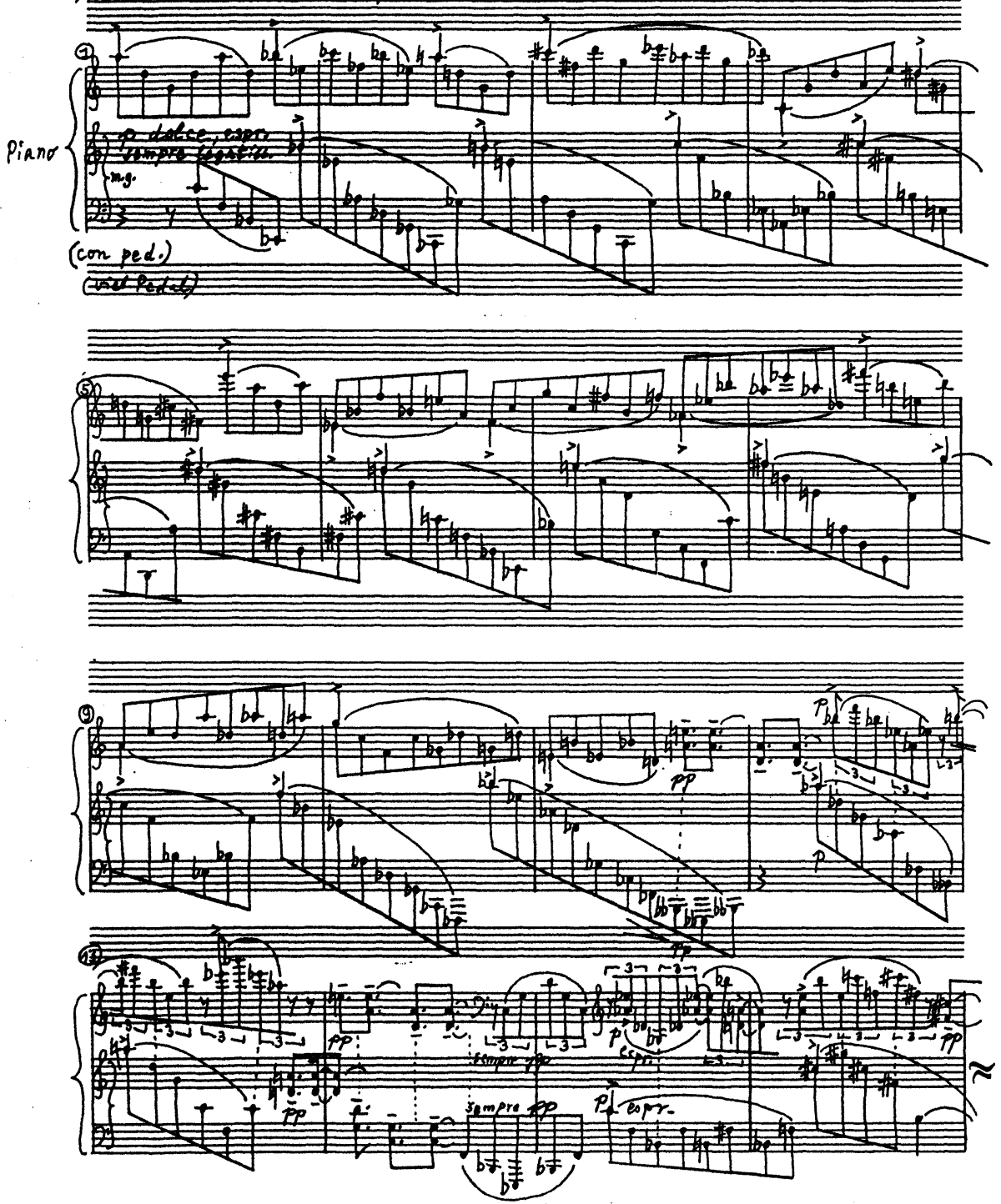
Ex. 13 : Étude Pour les arpèges composés de Debussy (mesures 1-6).

XI POUR LES ARPÈGES COMPOSÉS
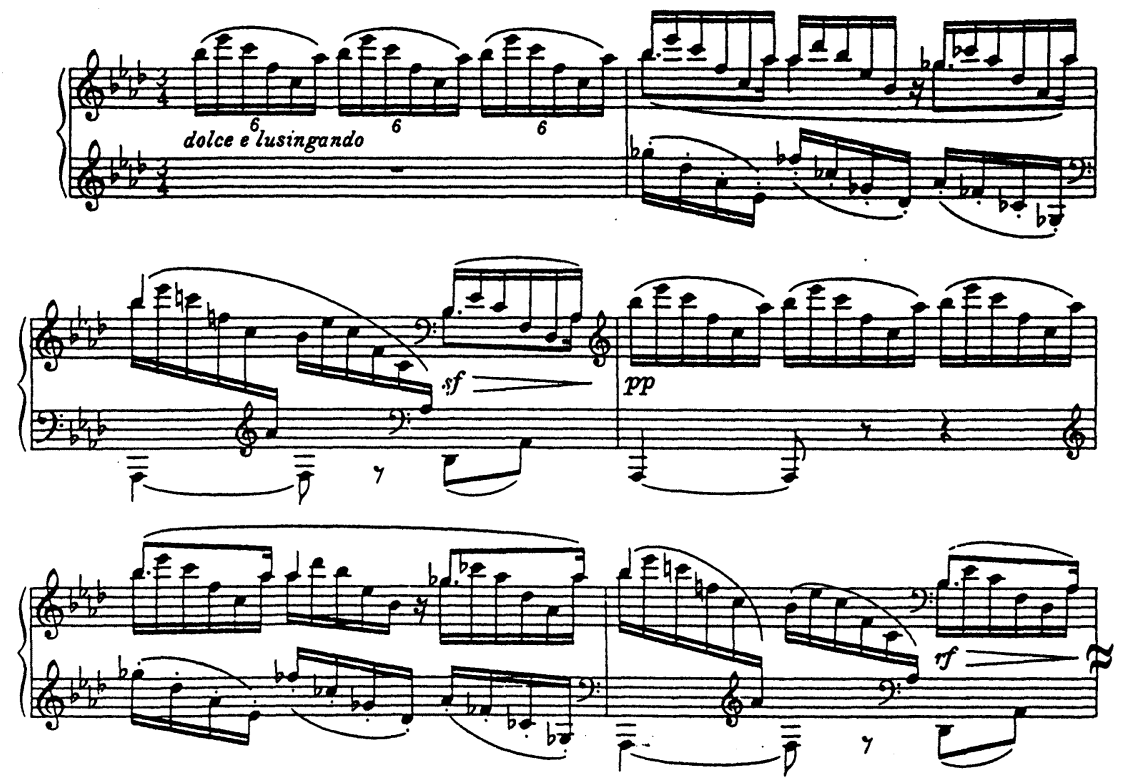
Es. 14: Touches bloquées, de Ligeti (lère page)
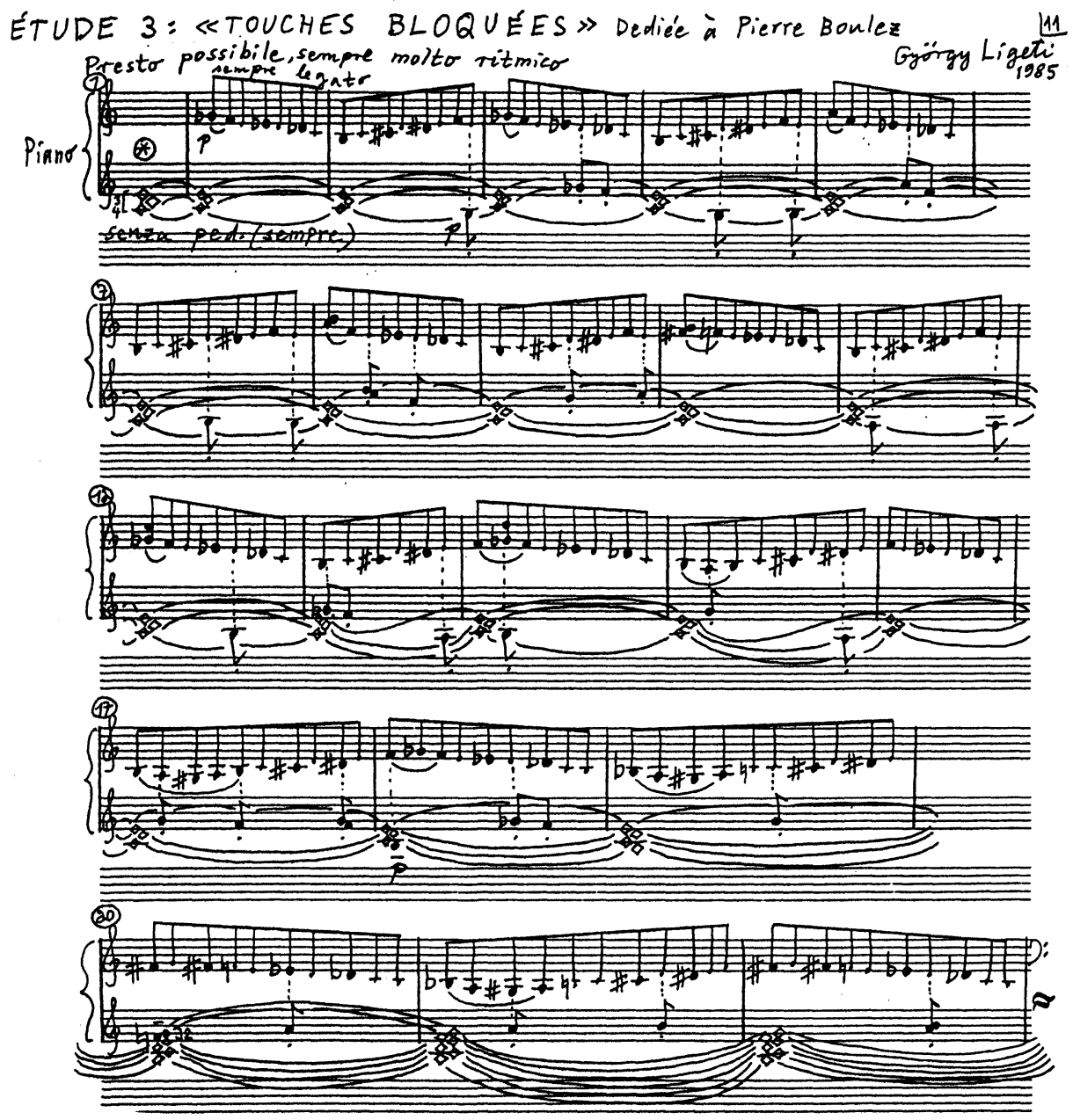


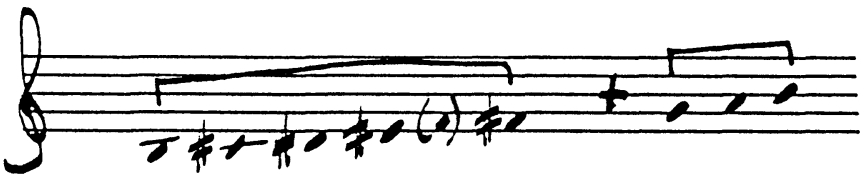

Ex. 15 : Touches bloquées, mode de départ (pentacorde lydien + terminaison phrygienne)

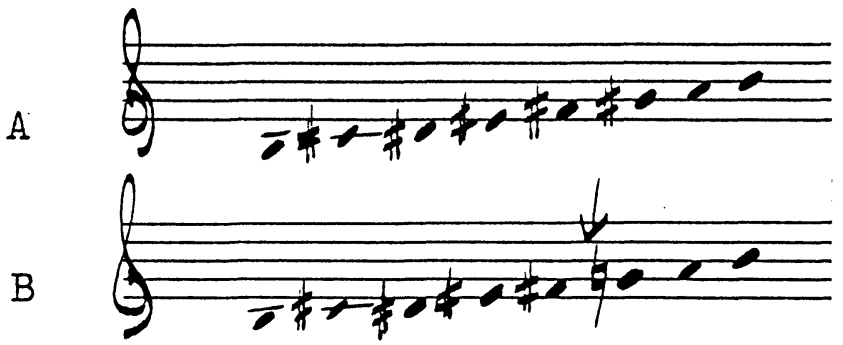

Ex. 16 : A, "mode acoustique" (Bartók) sur si B, mode de départ de Ligeti (Touches bloquées) 
Ex. 17 : Fanfares de Ligeti, lère page

ÉTUDE 4: "FANFARES" Dédiée à Volker Banfield

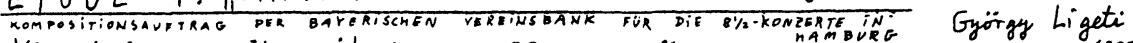

Vivacissimo molto ritmico os 63, con allegria e clabaió 1985

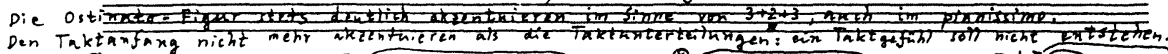

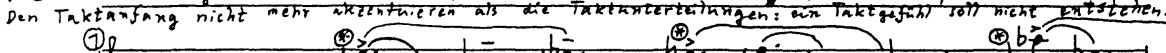

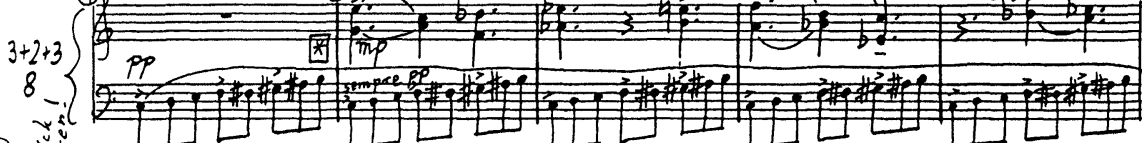

ข)

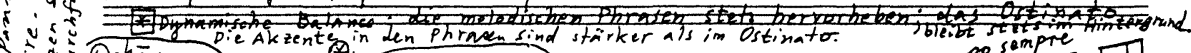

is

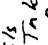

is

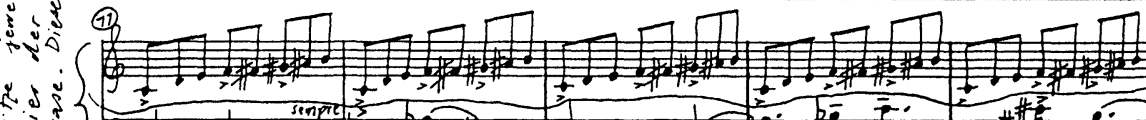

L.

is $\}$

in

5xin

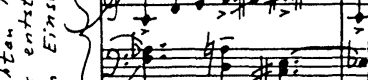

iับ

$=4$

$4+17+17$

$>$ pp sempre

5.

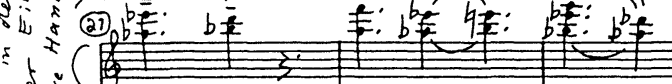

\&ेน

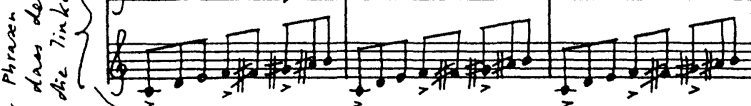

\$: is

1.

类

की

sin

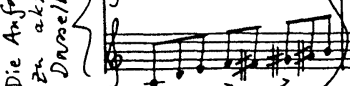

(4) :

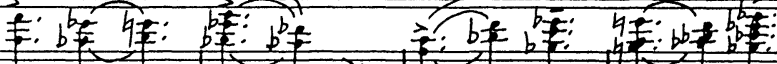



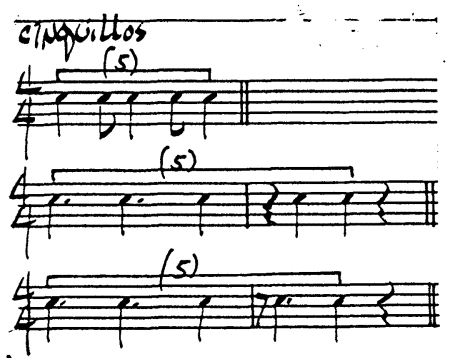

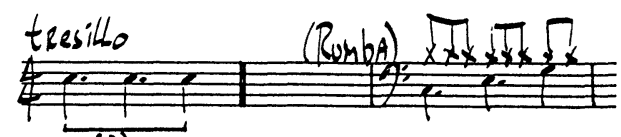

(3)
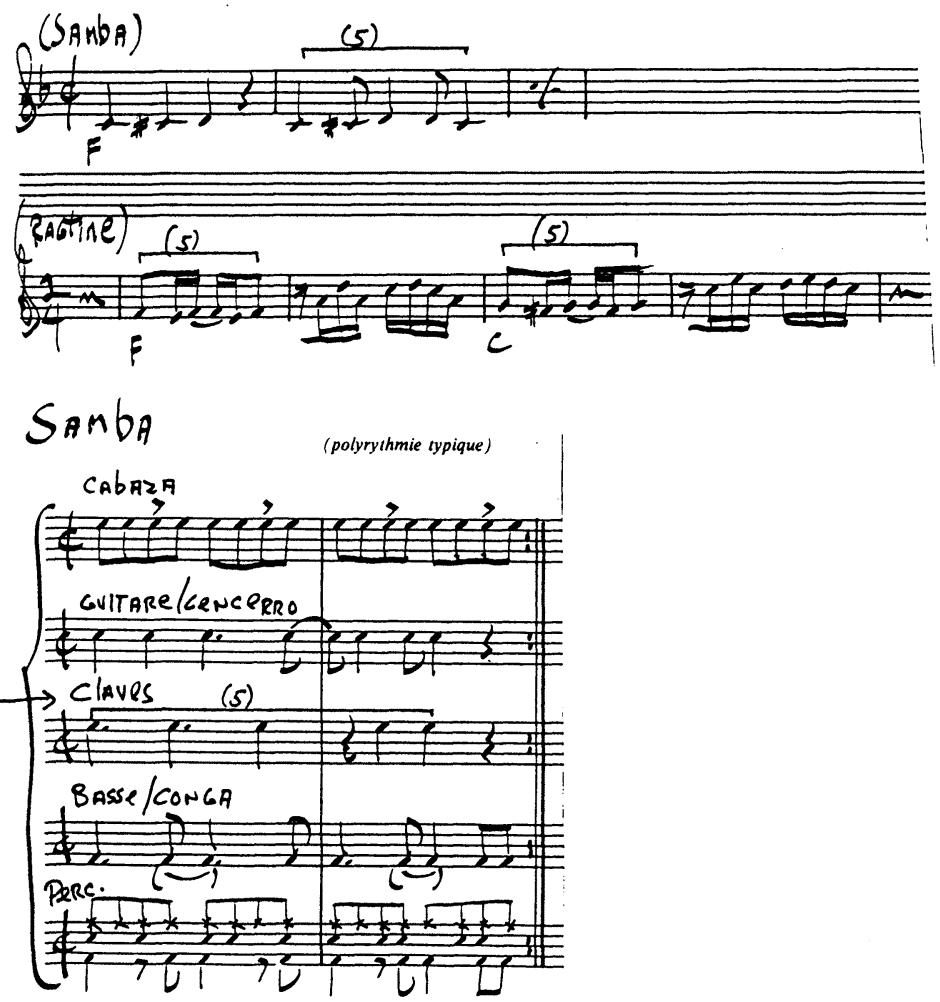

Ex. 18 : Articulation asymetrique en "tresillo" et "cinquillo" typique, entre autres, de la Rumba ou de la Samba.

75 


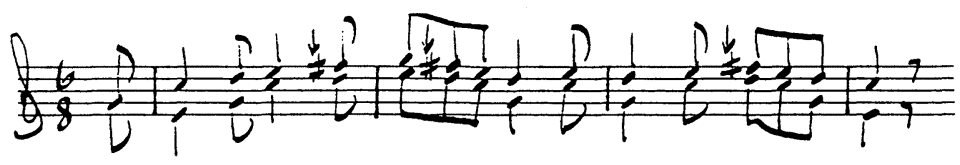

Ex. 20 : Fanfares de Ligeti, Sonneries de cuivres stylisées (mes. 86-95).
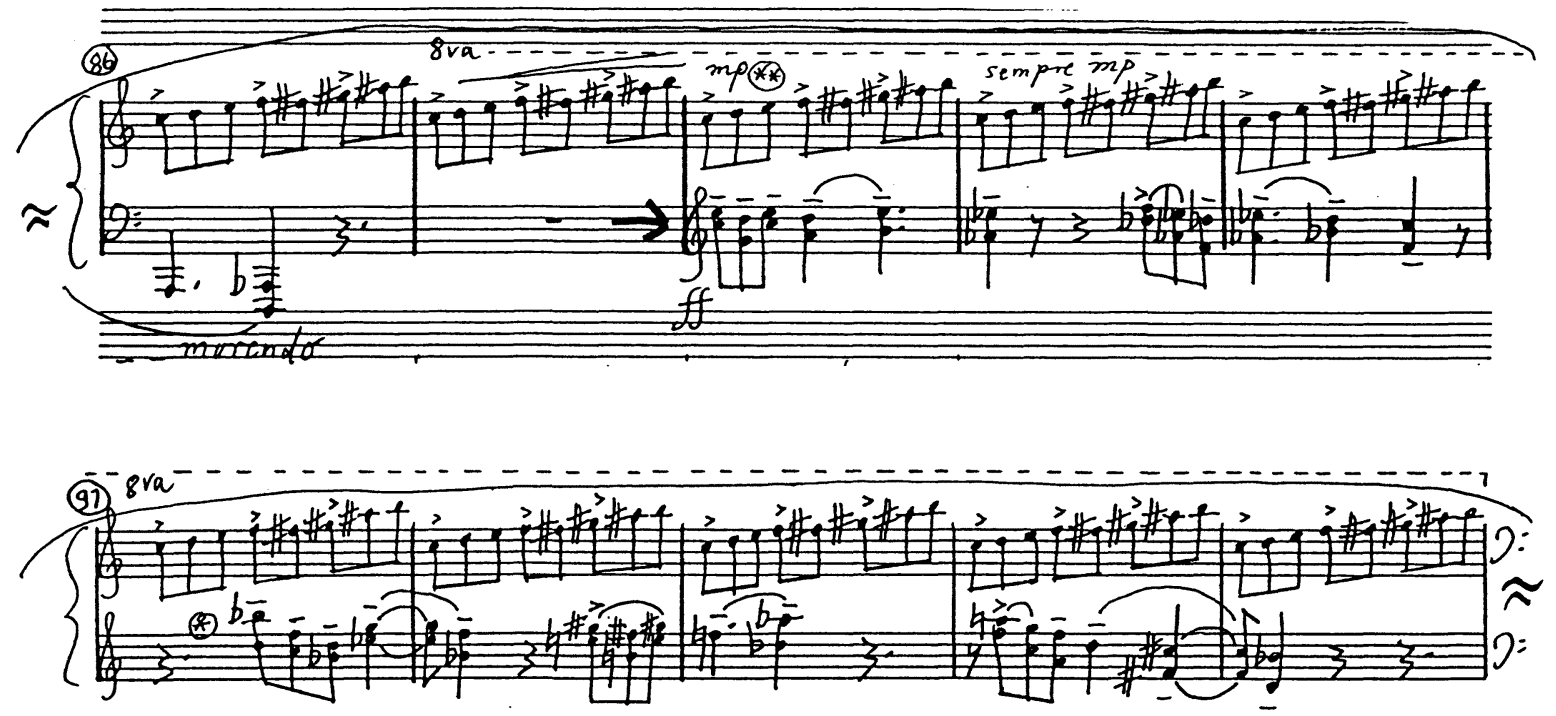

By permission of the Ligeti Publisher, B. Schott's Söhne Mainz. 
Ex. 21 : Arc-en-ciel de Ligeti (lère page)

ETUDE $5:$ "ARC-EN-CIEL" kompositionsavetrag der a varerischen véreins-

Dédiée à Louise Sibourd

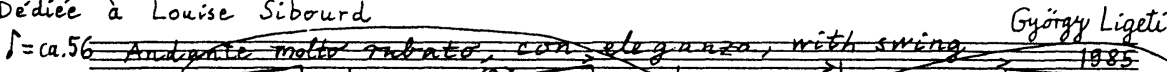
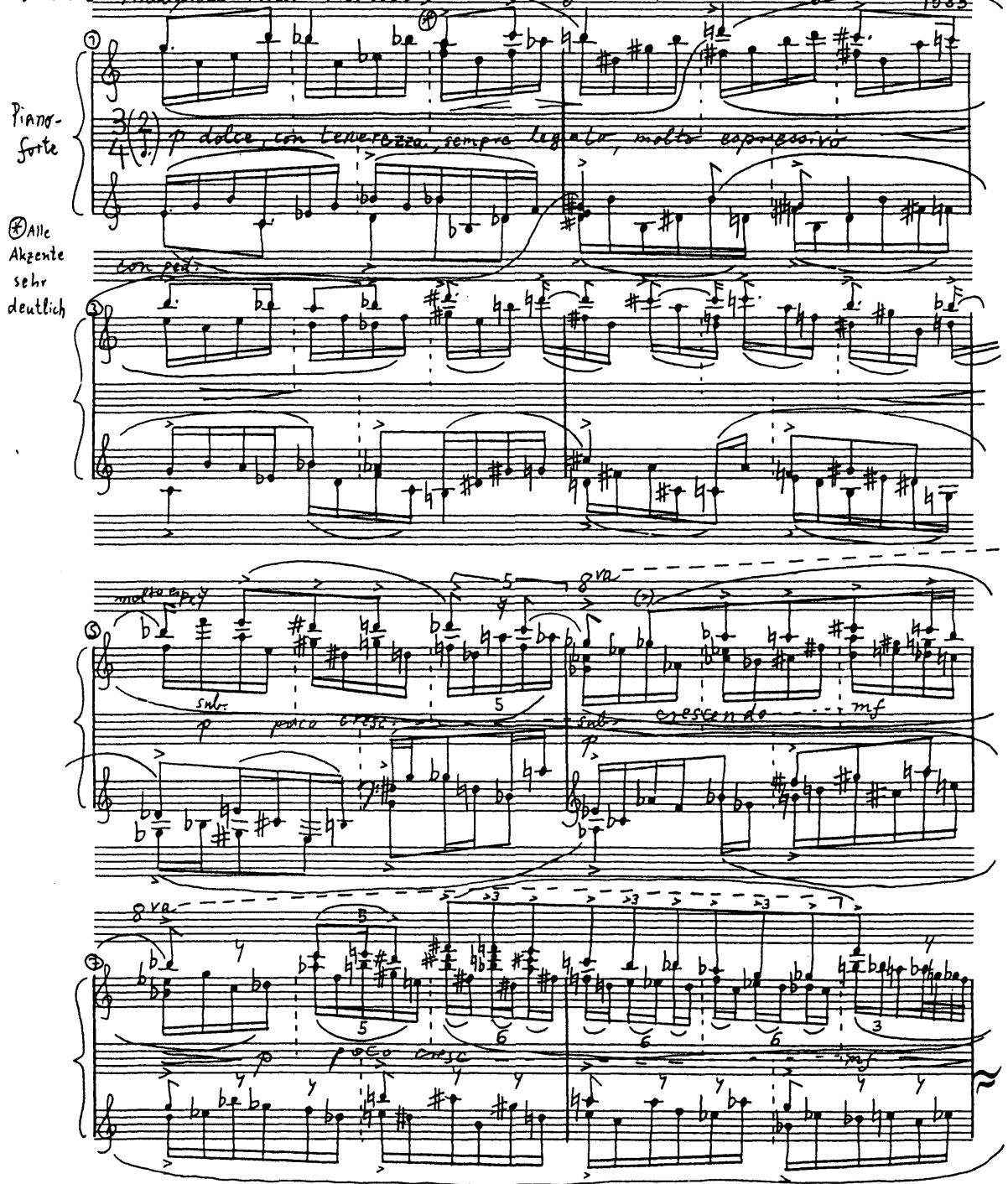

By permission of the Ligeti Publisher, B. Schott's Söhne Mainz. 
Ex. 22 : Des Abends de Schumann, lère section

$$
\begin{gathered}
\text { Des Abends } \\
\text { Le soir - Evening }
\end{gathered}
$$

Sehr innig zu spielen (komponiert 1837)
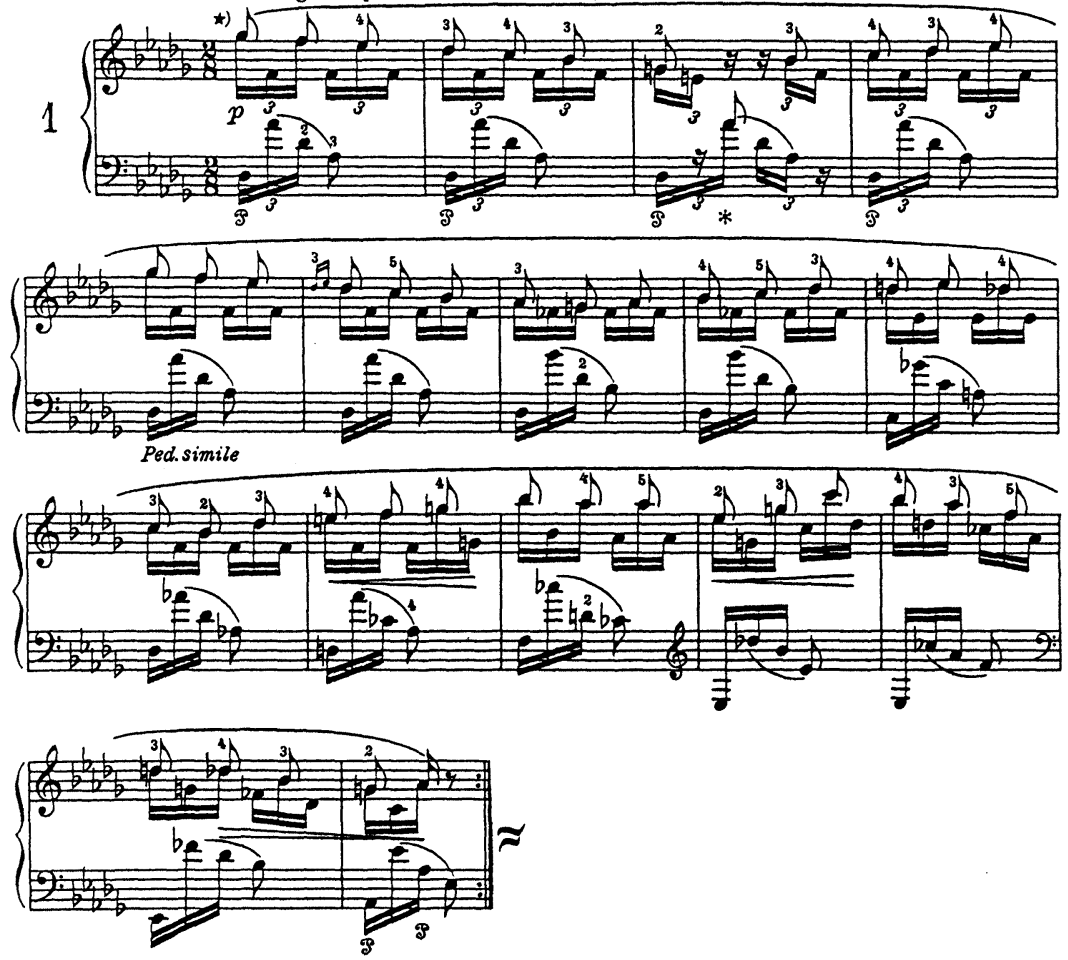
Ex. 23 : Nirvana de Herbie Mann, thème

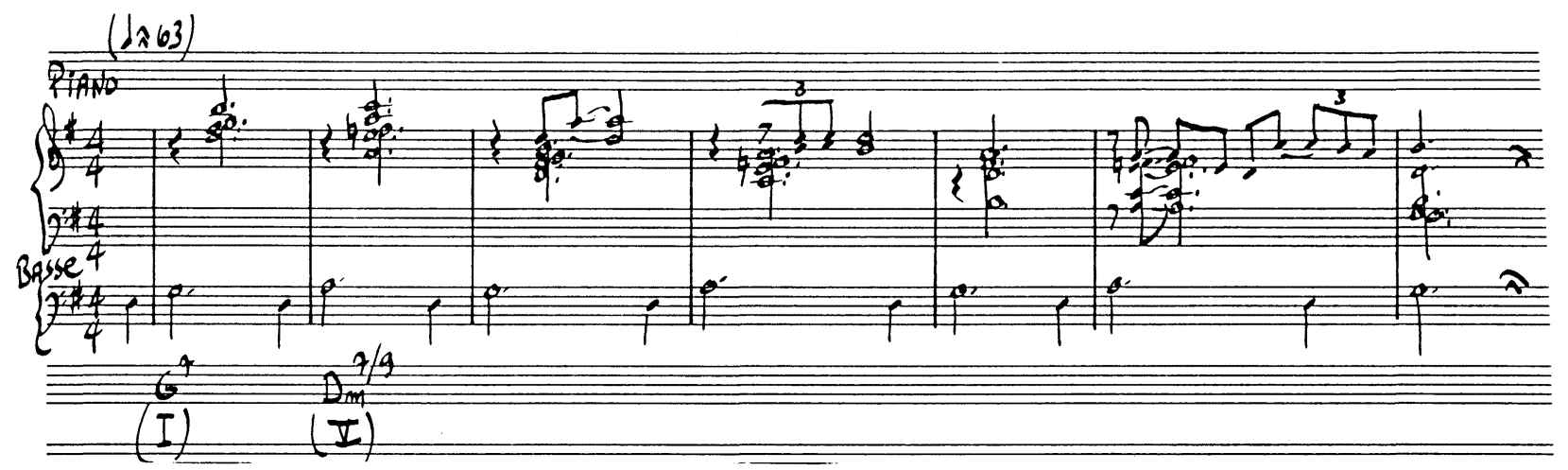

Ex. 24 : Arc-en-ciel de Ligeti, progression d'accords (mesures 1-7)

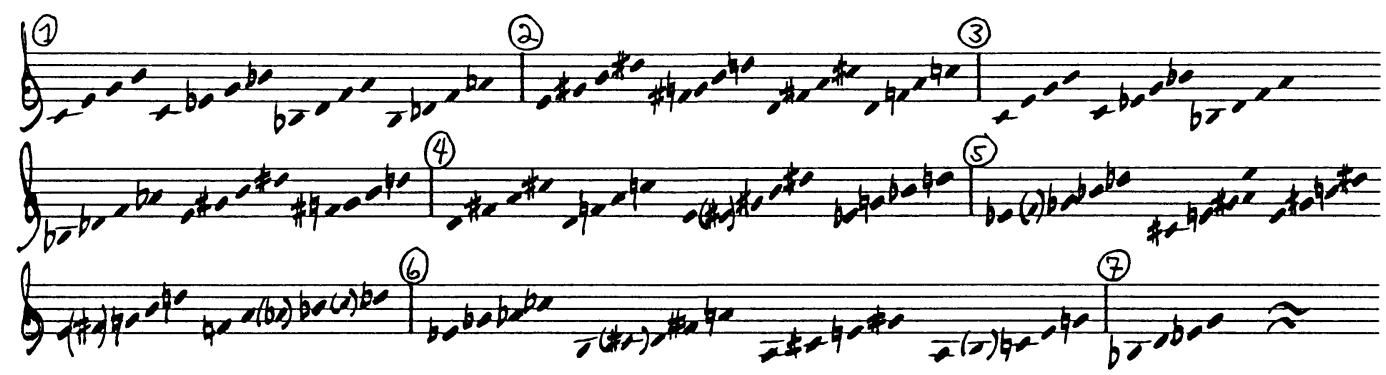


Ex. 25 : Automne à Varsovie de Ligeti, motifs "Lamento" tirés de la lère page de la partition.

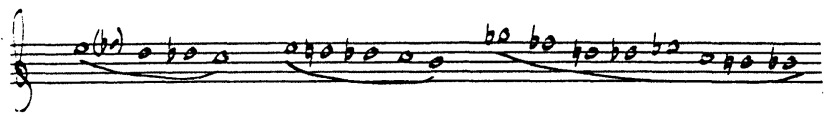

Ex. 26 : Cintecul bradului, chant funéraire - roumain - de Transylvanie.

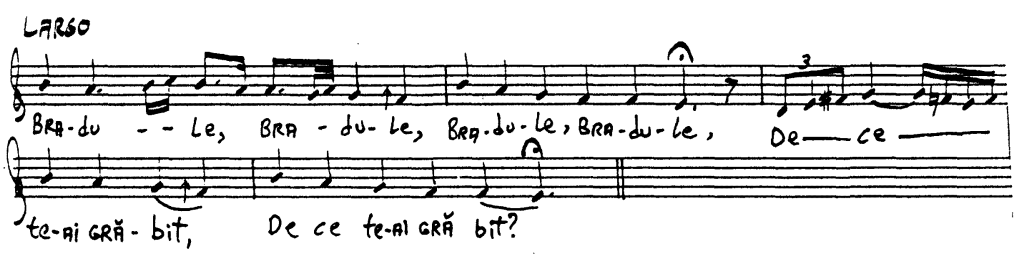

Ex. 27 : Motif "Lamento" tiré de Didon et Enée de Purcell.
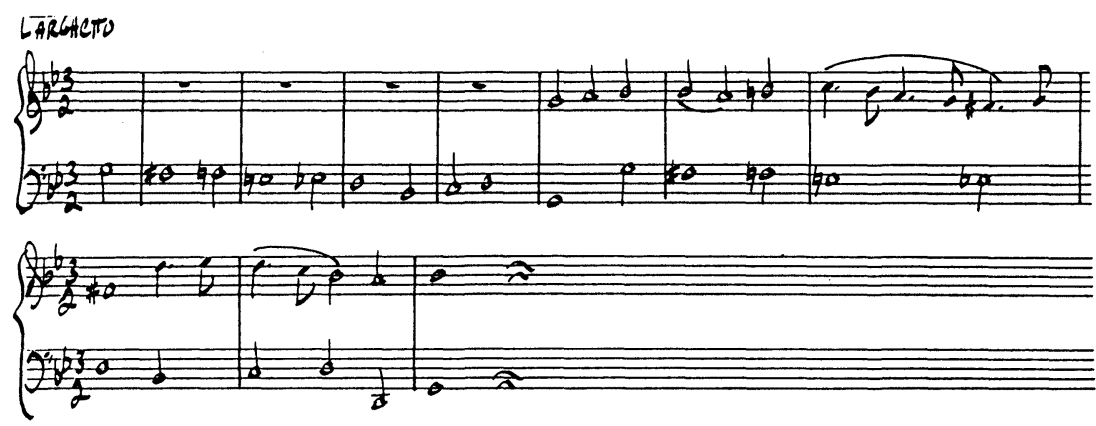
Ex. 28 : Automne à Varsovie de Ligeti (mes. 18, 19, 20)
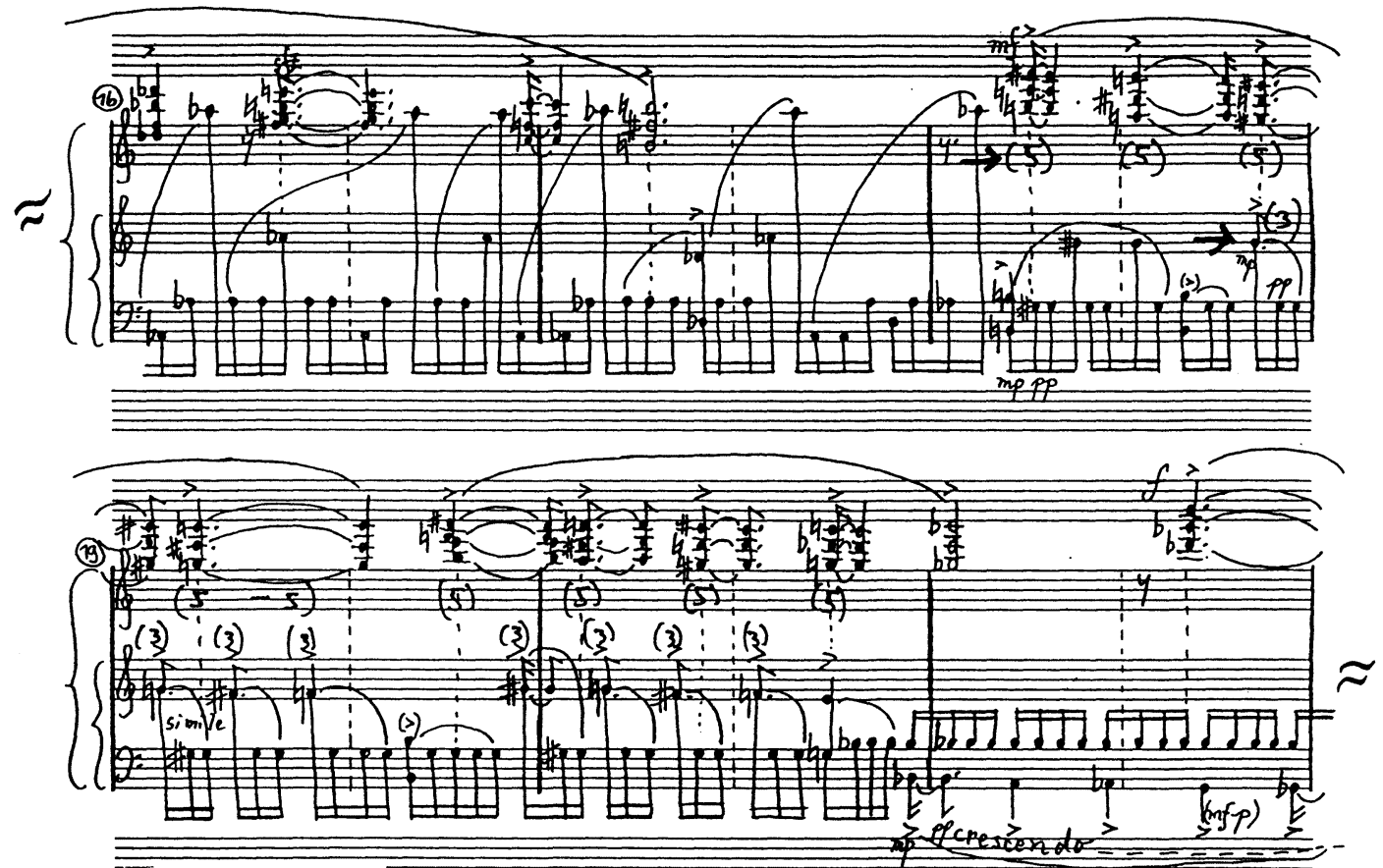
Ex. 29 : Automne à Varsovie, (mes. 76-82), superposition de 3 puis 4 "tempi"
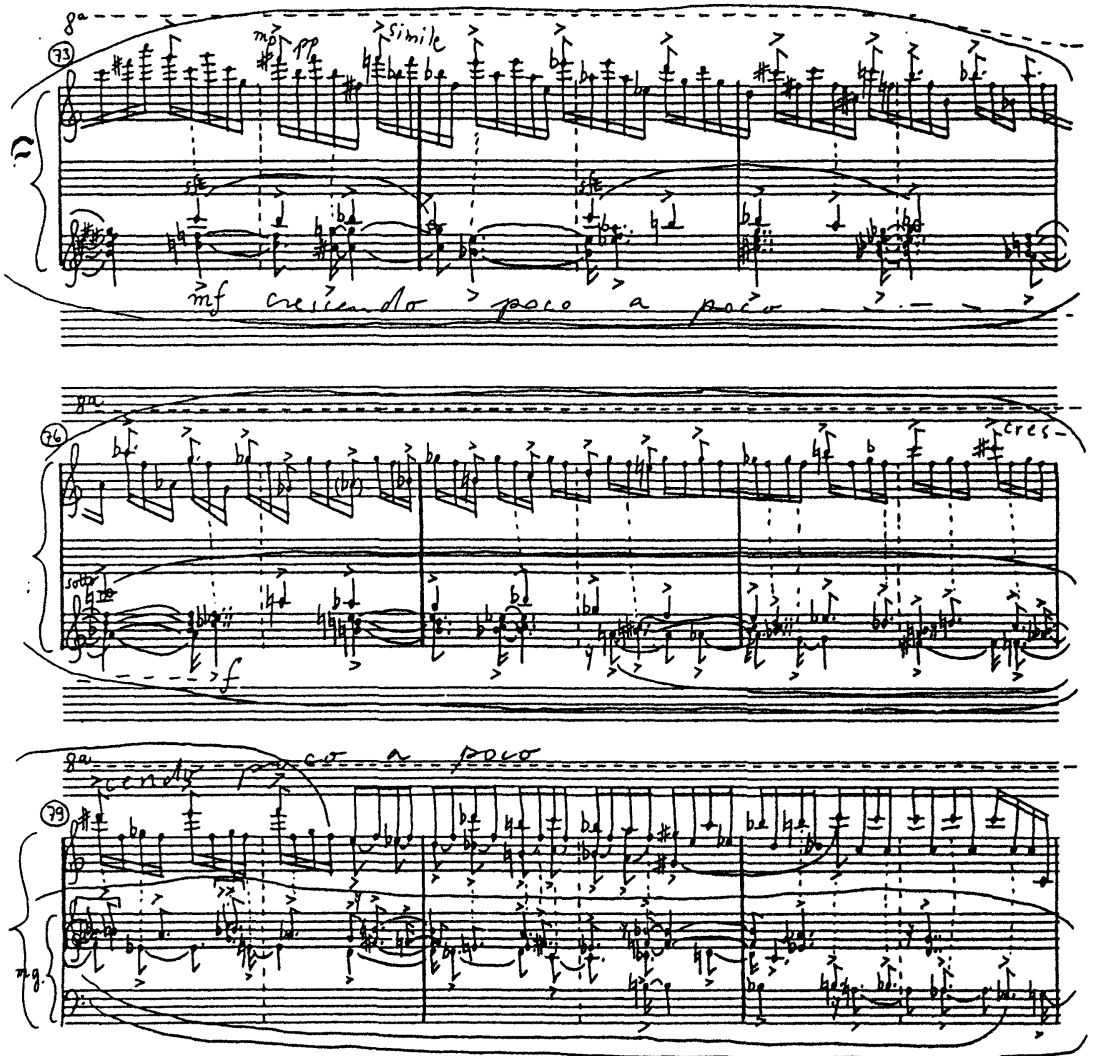

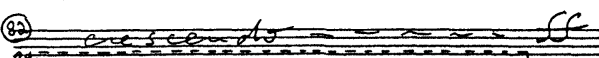

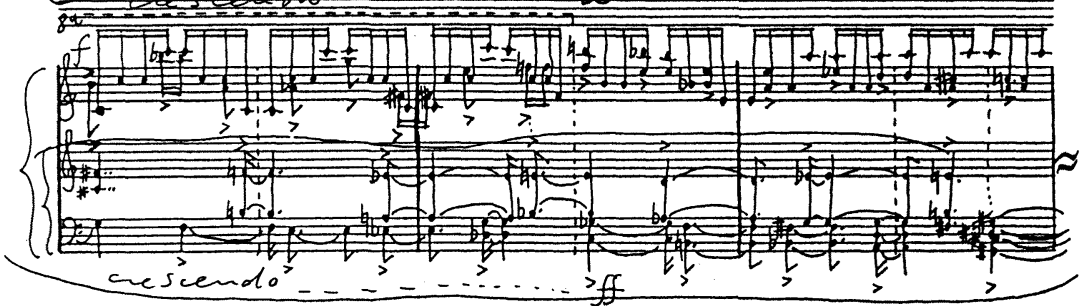

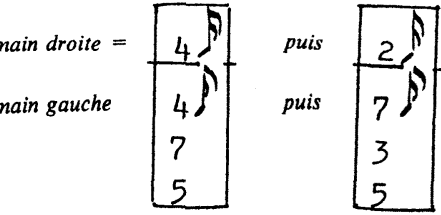

By permission of the Ligeti Publisher, B. Schott's Söhne Mainz. 
Ex. 30 : Étude op. 10 no 10 de Chopin (début)
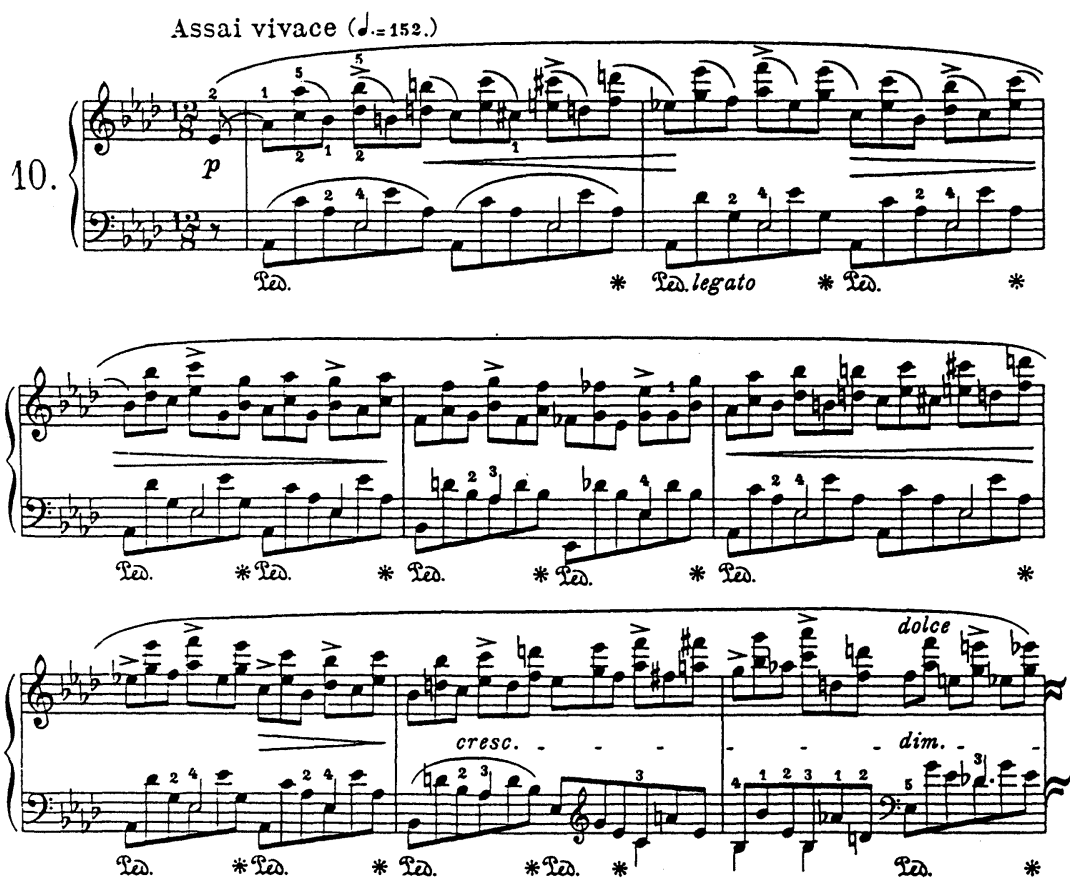

Superposition main droite $2+2+2$

main gauche $3+3$ 